\title{
Landmarks and new frontiers of computational fluid dynamics
}

Joseph J. S. Shang(D)

Correspondence: joseph.shang@ wright.edu

Wright State University, 3640

Colonel Glenn Highway, Dayton,

$\mathrm{OH}$ 45435-0001, USA

\begin{abstract}
A narrative of landmarks in computational fluid dynamics (CFD) is presented to highlight the cornerstone achievements. Illuminating accomplishments starting from the very beginning of the coherent development until the most recent progress will be elucidated over the span over more than six decades. Meanwhile, the cuttingedge scientific innovations will also be discussed for their lasting impacts to fluid dynamics and the physics-based modeling and simulation discipline. To traverse such a vast domain over time by a single presentation, numerous and excellent contributions to CFD will be unavoidably overlooked. Nevertheless it is my ardent hope that the present discussion will be able to reaffirm excellence in research and to identify new frontiers for scientific research. Especially, the challenges to future innovations will also be delineated to recommend for potential and fertile research areas for the modeling and simulation science.
\end{abstract}

Keywords: Computational fluid dynamics, Numerical algorithms, Turbulence, Interdisciplinary computational fluid dynamics

\section{Introduction}

In order to discuss the physics-based modeling and simulation discipline, the underlying principles must be explicitly stipulated to define its limitations. The traditional computational fluid dynamics (CFD) technique is mostly applying in the continuum gas domain which is limited to the negligible Knudson number; $N_{n}=\lambda / l<<1.0$. In this physical domain, the mean-free-path of particle collisions is negligible in comparison with the characteristic length of the flowfield considered. In the continuum regime, the compressible Navier-Stokes equations become the governing equation for describing fluid dynamics in the macroscopic scale, and the nonlinear partial differential equations system is the incompletely parabolic type. Even though the incompressible Navier-Stokes was known to us as far back as 1827, only more recently the system of equations was derived in integral form via the control-volume formulation to become the basis for the finite-volume approach. Nevertheless, the necessary initial values, boundary conditions, and their placement and implementation are mandatory to achieve a unique numerical simulation.

Numerical algorithms are inseparable parts of CFD research, also are the most demanding and creative efforts of this discipline, because they dictate the computational accuracy that provides the required physical fidelity to any computational simulations. In the gist, the numerical algorithm and the computational procedure research is a tool

(c) The Author(s). 2019 Open Access This article is distributed under the terms of the Creative Commons Attribution 4.0 International License (http://creativecommons.org/licenses/by/4.0/), which permits unrestricted use, distribution, and reproduction in any medium, provided you give appropriate credit to the original author(s) and the source, provide a link to the Creative Commons license, and indicate if changes were made. 
development endeavor. The type of research is subjected to the most rigorous scrutinizing for consistency, uniqueness, and stability issues in numerical analysis. For this reason, the value of a numerical algorithm always rises and falls according to practical problem solving needs. The adopted numerical algorithms at the early stage of CFD development are mostly explicit schemes for their simplicity in programming and limiting by the computer memory. The widen CFD application demands have driven computer technology from scalar to vector and finally to concurrent data processing, meanwhile the unconditionally stable implicit schemes also reached maturate. When CFD expanded to all flow regimes from subsonic, transonic, supersonic, to hypersonic; the need for treating piecewise continuous numerical solutions is paramount and the level of sophistication also elevated. There are simply too many numerical algorisms to be completely and precisely discussed, as the consequence, only the classic results that have withheld the test of time are included together with the most recent progress in high-resolution procedures.

The landmarks for CFD accomplishments are presented according to their contributions to scientific discovery in fluid dynamics and technical breakthrough to aerospace engineering. The brief review starts from a very few fundamental concepts which lead to a coherent development during WWII in the middle of 1940s. Followed a remarkable growing period initiate by NASA (National Aeronautics and Space Administration) research centers in the later 1960s, the pursuit of scientific excellence was firmly instilled in this technical discipline. In the subsequence years, CFD expands into interdisciplinary arenas for combustion, propulsion, structure dynamics, flight control, thermal protection for earth reentry space vehicles. The matured technology has opened avenues for aerospace vehicle design and analysis; it is self-evident that CFD was widely used for the Space Shuttle design and evaluation, and the National Aerospace Plane (NASP) was entirely designed by CFD techniques. Shortly afterwards, the CFD techniques are transferring into the computational electromagnetics and computational magnetohydrodynamics disciplines.

The arriving of concurrent, high performance super computational technology provides an extraordinary opportunity for CFD to create many new science frontiers. The first and the straightforward opportunities are to address the most challenged and the least understood fluid dynamics phenomena such as the bifurcation, hysteresis, and turbulence. Based on the kinetic theory of gas, these fluid dynamic phenomena are addressable by direct numerical simulation with accurate initial values and boundary conditions, without imposing any statistical ensemble approximations. The second and greater challenge is expanding the scientific basis for simulating high enthalpy or high temperature gas phenomenon by removing the elastic collision restrictions from kinetic theory of gas; namely the internal degree of freedom in vibrational and electron excitations of atom and molecule will be described by inelastic collisions involving quantum mechanics. Based on our accumulated knowledge, these opportunities and possible new approaches will be discussed and outlined.

\section{Governing equations}

The governing equation for traditional CFD in continuum domain is the time-dependent, compressible Navier-Stokes equations, which first published in 1827 for incompressible flows [1]. The closure of the nonlinear partial differential equations system was achieved 
by Stokes through the relationship between the bulk and molecular viscosity coefficients [2]. In the strict sense, the governing equations are applicable only to the Newtonian fluid for which the shearing stress is linearly proportional to the rate of strain. In essence, the Navier-Stokes equations describe gas particle dynamics on the macroscopic scale. The system of equations is germinated from the kinetic theory of gas [3]: Within a given dynamic system, the gas particles always move in random motion with the kinetic and potential energy of their own. The individual particle's behavior in microscopic scales can only be meaningfully described through statistic means. Based on the probability theory, it introduces a weighing factor known as the distribution function, $f\left(x_{i} c_{i} t\right)$ in the six-degree-of-freedom geometric and velocity space or the phase space. By the definition of Hamiltonian that a system of particle possesses kinetic and potential energy, the rates of change have the symmetric property relating to the particle velocity and geometric position in the phase space. According to the Liouville's theorem, the number density of a dynamic system of moving particles in the phase space must remain constant. The rate of changes for the distribution function of the particles is governing by the Boltzmann or Boltzmann-Maxwell equation of the distribution function,

$$
\partial f\left(x_{i}, c_{i}, t\right) / \partial t+c_{i} \cdot \nabla f\left(x_{i}, c_{i}, t\right)+F_{i} \cdot \nabla_{u} f\left(x_{i}, c_{i}, t\right)=\left[\partial f\left(x_{i}, c_{i}, t\right) / \partial t\right]_{c}
$$

where $c_{i}$ and $x_{i}$ are the specular velocity and the position of particles in the phase space. The external force exerting on each particle is designated as $F_{i}$. The integro-differential equation is very difficult to solve, and the solutions of the Boltzmann equation in term of probability is also not suitable for engineering applications.

In order to simplify the Boltzmann equation, the particles dynamics contributed by the collision integral on the right-hand-side of the Maxwell equation are simplified by a group of elastic spheres. This simplification makes the total energy of particle's internal degree of freedom an invariant; in other words, the internal excitations of the particles are neglected. As the consequence, the inter-atomic and inter-molecular excitations and energy cascading between internal modes of gas particles are not considered. In an addition, the interaction of particles is limited to binary encounters. The binary dynamic exchange by elastic collision actually establishes the concept of collision equilibrium condition, leading to:

$$
\left[\partial f\left(x_{i}, c_{i}, t\right) / \partial t\right]_{c}=\iiint\left[f\left(c_{i}^{\prime}\right) f\left(x_{i}^{\prime}\right)-f\left(c_{i}\right) f\left(x_{i}\right)\right]\left(c_{i}^{\prime}-c_{i}\right) d^{3} x_{i} d^{3} c_{i}
$$

Under the dynamic equilibrium condition;

$$
f\left(c_{i}^{\prime}\right) f\left(x_{i}^{\prime}\right)=f\left(c_{i}\right) f\left(x_{i}\right)
$$

The link between the microscopic and macroscopic description of gasdynamics can be established by the method of moments, but the most successful approach is the Enskog's infinite series expansion [3]. Under the collision equilibrium condition, the Boltzmann equations transform directly to the Euler equations, which are essentially the Navier-Stokes equations but containing only the inviscid terms. The hierarchy of fluid dynamics governing equation is depicting in Fig. 1.

Under the nonequilibrium collision condition, the transport properties of the gas must be included. To be consistent with theoretic formulation, the transport properties of gas are obtained by the gas kinetic theory of diluted gas mixtures, and it is a 


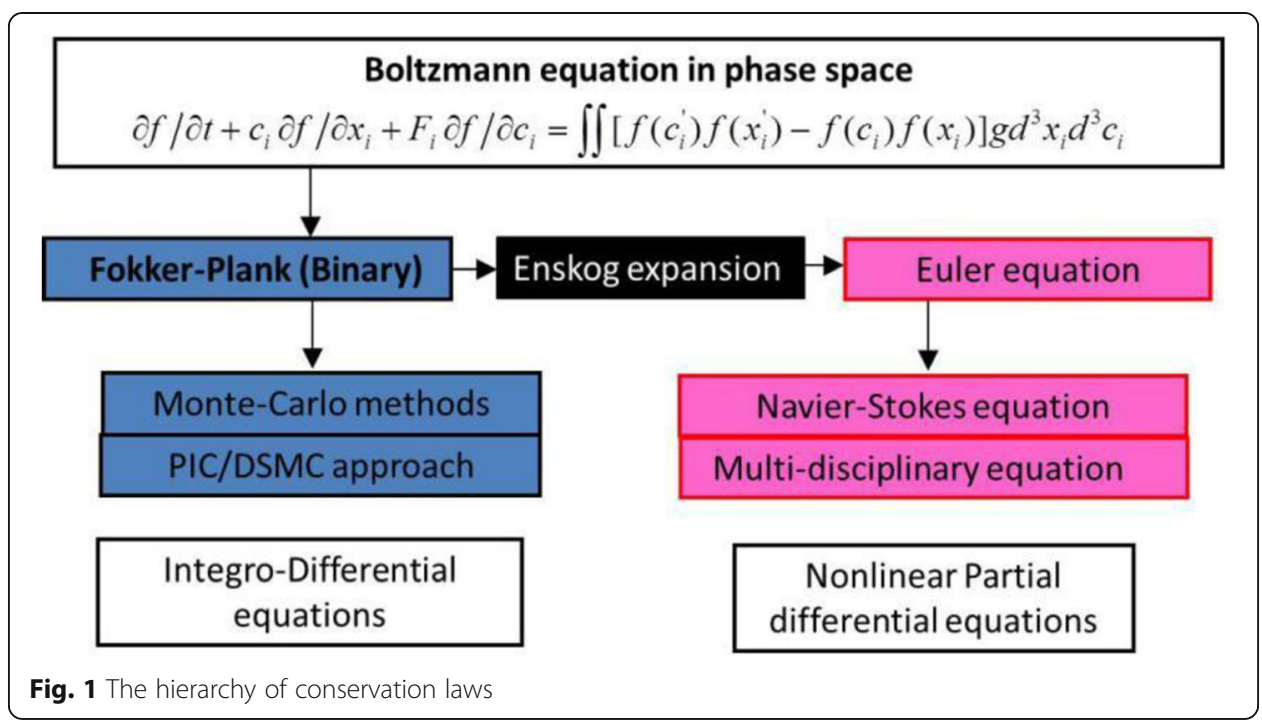

landmark achievement by the kinetic theory of gas [4]. The transport property of any combination of gaseous mixture is derivable by the inter-molecular potential function. The required collision integrals and cross sections for the gas molecular viscosity, thermal conductivity, and binary diffusion coefficients of individual species have been obtained by the Lenard-Jones potential for gas molecules [5].

The diffusion coefficient of a binary gas mixture is;

$$
D_{i, j}=1.858 \times 10^{-3} \sqrt{T^{3}\left(M_{i}+M_{j}\right) / M_{i} M_{j}} / \sigma_{i, j}^{2} \Omega^{(1,1)}
$$

The molecular viscosity of a single species is given as.

$$
\mu=2.67 \times 10^{-5} \sqrt{M_{i} T} / \sigma_{i}^{2} \Omega^{(2,2)}
$$

and the thermal conductivities for a mono-atomic and poly-atomic molecules are;

$$
\begin{aligned}
& \kappa_{i, m}=1.989 \times 10^{-4} \sqrt{T / M_{i}} / \sigma_{i}^{2} \Omega^{(2,2)} \\
& \kappa_{i, p}=2.519 \times 10^{-4} \sqrt{T / M_{i}} / \sigma_{i}^{2} \Omega^{(2,2)}
\end{aligned}
$$

The collision integrals $\Omega^{(1,1)}, \Omega^{(2,2)}$ and the transport cross section $\sigma_{i}$ are obtained by performing three consecutive integrations of the inter-molecular potential function: The integrations are performed first to determine the classic deflection angle as the impact parameter, then from the impact parameters to get the relevant cross section. Finally, an averaging process is carried over the entire range of energy to produce the collision cross section as a function of temperature [6].

For an inhomogeneous gas mixture, the transport property can be approximated by the Wilke's mixing rule [5]. However, in most practical engineering applications, the transport properties of air by kinetic theory of gas are replaced by empirical formulations and similarity aerodynamic parameters such as the Prandtl number. Whence the transport properties are known, the time-dependent, three-dimensional, compressible Navier-Stokes equations can be given as; 


$$
\begin{aligned}
& \frac{\partial \rho}{\partial t}+\nabla \cdot(\rho u)=0 \\
& \frac{\partial \rho u}{\partial t}+\nabla \cdot(\rho u u-\tau)-\rho f=0 \\
& \frac{\partial \rho e}{\partial t}+\nabla \cdot(\rho e u+q+u \cdot \tau)-\rho(f \cdot u)=0
\end{aligned}
$$

The system of equations is also known as the conservation laws. Equation (5-a) is often referred to as the continuity equation which is the most fundamental concept of Newtonian mechanics in that the mass and energy is not exchangeable like in quantum mechanics. The conservation of momentum equation, Eq. (5-b) is the only vector equation in the system, and it is the Newton's second law of motion with a possible external force $f$, such as gravitation or the electromagnetic force. The nonlinear transfer of momentum by convection is represented by a dyadic, $\rho u u$, which is the principal component of the inviscid terms. In fact, it is also the source of turbulence from vortex interactions within the entire flow field.

The shear stress term, on the other hand, is another second rank tensor described as;

$$
\tau=(-p+\lambda \nabla \cdot u) I+\mu \operatorname{def}(u)
$$

where, $\lambda$ and $\mu$ are the bulk and molecular viscosity and $I$ is the identity matrix. The last term of the stress tensor is referred to as the deformation tensor;

$$
\operatorname{def}(u)=\nabla u+(\nabla u)^{T}
$$

The transpose operator of the gradient $u,(\nabla u)^{T}$ is simply by replacing the rows by columns in the matrix element of $\nabla u$. The deformation tensor has an important fluid mechanical interpretation in that the diagonal derivatives represent the longitudinal strain, while the off-diagonal derivatives represent the angular deformation of fluid motion. As the consequences, the viscous flow at the solid-fluid interface boundary produces shear stress. Whereas, the inviscid terms associated with the normal component of the stress tensor lead to expansion and compression of the flow.

Equation (5-c) is the conservation of energy law, it is just the second law of thermodynamics, and the internal energy is defined as;

$$
\rho e=\rho\left(\int c_{c} d T+u \cdot u / 2\right)
$$

The heat transfer term includes the Fourier's law for conductive, convective by different species with different diffusion velocity, and the radiation energy transfer;

$$
q=-k \nabla T+\Sigma \rho_{i} u_{i} h_{i}+q_{r a d}
$$

It is important to know that the system of equations, Eq. (5-a), (5-b), and (5-c) constitute a nonlinear, incompletely parabolic partial differential equation system [7]. Any unique solutions to the compressible Navier-Stokes equation must satisfy the compatible initial values and boundary conditions to the differential equations system.

It is interesting to realize that the widely adopted finite-volume formulation of Navier-Stokes equations in integral form via a control volume formulation was first formerly derived by Rizzi and Inouye [8]. The balancing of outward normal vector flux 
components across the control surface between adjacent control volumes becomes the only constraint to the flux vector splitting technique.

In practical applications, the conservation laws Eq.; (5-a), (5-b), and (5-c) are usually written in a strong conservation flux vector form. On the Cartesian coordinates, they appear as

$$
\partial U / \partial t+\partial F / \partial x+\partial G / \partial y+\partial H / \partial z=0
$$

where the dependent variables are; $U=U(\rho, \rho u, \rho v, \rho w, \rho e)$.

The Rankine-Hugoniot jump condition across a shock wave is recoverable from the Euler equations which constitutes the hyperbolic partial differential equations. Therefore, the conservation laws are often solved separately but concurrently for the inviscid and viscous terms. For this reason, the flux vectors $F, G$, and $H$ are often split into components of inviscid and viscous terms as;

$$
\partial U / \partial t+\partial\left(F_{i}+F_{v}\right) / \partial x+\partial\left(G_{i}+G_{v}\right) / \partial y+\partial\left(H_{i}+H_{v}\right) / \partial z=0
$$

For simulating complex configurations, the flux vector formulation is often transformed onto a generalized curvilinear, body oriented coordinate by means of the chain rule of differentiation [9]. Again through metric identities of coordinate transformation, the equation can still be rewritten in the strong conservation form.

\section{Numerical algorithms evolution}

The numerical algorithm is the heart of computational fluid dynamic, because it is the necessary translator between numerical analysis for fluid dynamics via computers. The history of CFD is also ultimately related to the development of programmable digital computers: In 1833 Charles Baggage originated the idea of a programmable computer, but the first patent for the ENIAC computer (electronic numerical integrator and computer) was recorded in 1947 for the truly programmable computer using transistors.

The interrelation between numerical algorithms and computational results is depicted by a graphic presentation in Fig. 2. The illustrated algorithm is the diminishing residue return (DRR) scheme; the right-hand-side of the conservative law represents the physics

$$
\begin{gathered}
\frac{\partial \boldsymbol{U}}{\partial t}+\frac{\partial \boldsymbol{F}}{\partial x}+\frac{\partial \boldsymbol{G}}{\partial y}+\frac{\partial \boldsymbol{H}}{\partial z}=0 \\
\left.\frac{\partial \boldsymbol{U}}{\partial t}+\left(\frac{\partial \boldsymbol{F}}{\partial \boldsymbol{U}}\right)\left(\frac{\partial \boldsymbol{U}}{\partial x}\right)\right]+\left[\left(\frac{\partial \boldsymbol{G}}{\partial \boldsymbol{U}}\right)\left(\frac{\partial \boldsymbol{U}}{\partial y}\right)\right]+\left[\left(\frac{\partial H}{\partial \boldsymbol{U}}\right)\left(\frac{\partial \boldsymbol{U}}{\partial z}\right)\right]=0
\end{gathered}
$$

Discretize to get

$$
\begin{gathered}
\frac{\partial \boldsymbol{U}}{\partial t}=\frac{1}{\Delta t}\left[\boldsymbol{U}^{n+1}-\boldsymbol{U}^{n}\right]=\frac{\nabla \boldsymbol{U}^{n+1}}{\nabla t} \\
\left\{I+\Delta t\left[\frac{\delta}{\delta x}\left(\frac{\delta F}{\delta U}\right)+\frac{\delta}{\delta x}\left(\frac{\delta G}{\delta U}\right)+\frac{\delta}{\delta x}\left(\frac{\delta H}{\delta U}\right)\right]\right\} \Delta U^{n+1}=-\Delta t\left[\frac{\partial F}{\partial x}+\frac{\partial G}{\partial x}+\frac{\partial H}{\partial x}\right]
\end{gathered}
$$

\section{$[$ Numerics $] \Delta \boldsymbol{U}_{i, j, k}^{n+1}=[$ Physics $]$}

Fig. 2 Relationship between physics fidelity and numerical algorithm 
to be simulated. The left-hand-side of the equation is the numerical process and its sole purpose is keeping a stable computation. In fact, the illustration also implies the equivalent principle held for which if a stable numerical algorithm leading to a converged asymptote, the numerical result is ensured to be the unique solution.

There are two entirely different concepts for CFD formulations, and the most widely adopted approach is the Eulerian frame of reference. In this formulation the fluid dynamics is analyzed in a control volume fixed in space. Whereas, the Lagrangian approach is analyzing fluid dynamics by following a group moving gas particles in an enclosed control volume. The well-known direct simulation Monte Carlo (DSMC) method is built on the Largangian formulation, together with the particle-in-cell (PIC) method by Harlow [10]. For the PIC method, the fluid dynamics is represented by Lagrangian mass particles within a control volume. At each time step, the calculated internal energy and velocity are obtained and the conservation properties are checked by the sum of these final values before the process advances to the next time level. The DSMC and PIC methods have demonstrated to be well suited to study the time dependent and multi-spices fluid medium, and had been widely used for simulating rarefied gasdynamics and plasma dynamics [11].

The most predominant CFD algorithm pioneers are led by Richardson who introduced point iterative scheme to solve the elliptic partial differential equation as far back as 1910 [12]. Then Courant, Friedrichs and Lewy initiated the rigorous investigation procedure for examining the stability of a numerical algorithm by Fourier analysis in 1928. They also addressed the uniqueness and existence of the numerical results for partial differential equations [13]. It was Southwell who introduced a relaxation scheme to solve both the fluid dynamic and structure problem to become an accepted procedure for engineering application in 1940 [14]. Lax [15] and Godunov [16] addressed the most challenging and difficult issues in numerical analyses for resolving discontinuous fluid phenomena in a discrete space - the approximate Riemann problem. As it will be seen later, it remains to be the most studied problem in CFD.

In the early 1960s, the dominated numerical algorithms are mostly explicit schemes, such as the Lax-Wendroff, leap-frog, and fractional step methods for multi-dimensional problems [17]. When CFD ventures into increasingly complex fluid phenomena, the more efficient and stable implicit schemes are required. Especially, the ADI method $[18,19]$ has been effectively applied to all type of partial equations, except when applying to the time-dependent, three-dimensional hyperbolic system for which some forms of artificial dissipative terms must be appended to maintain computational stability. This shortcoming is removed by finite-volume formulation with an iterative solving scheme. In the subsequent developments, the basic ADI scheme has evolved into the strong implicit scheme (SIP) and the diminishing residual return (DRR) formulations. The matrix inversion procedures for ADI have also expanded to include the lower-upper (LU) decomposition technique, as well as, the line, Jacobi, Gauss-Seidel, conjugate relaxation procedures. In short, the ADI scheme still remains as the mainstay for most multi-dimensional solving procedure even to date [20].

The increasing demand of greater numerical efficiency and maintaining computational stability was met by the multi-grid approach [21]. The convergence rate of an iterative scheme has a close tie to the spectral radius of eigenvalues of the equation system and the residue error reduction process from the initial estimate. For the multi-grid approach, the 
data is processing through a sequence of different grid-point spacings in the computational domain to filter out the undesirable high frequency, unstable Fourier components from the solution. Another popular approach is adopting the unstructured grid technique to cluster cells where the high grid density is needed; the Delaunay Scheme [22] was introduced first for generating two-dimensional triangular and three-dimensional tetrahedron meshes. From the analytic geometry viewpoint, the pyramidal control volume topology is natural to uniquely define the control volume. The numerical results of an unstructured grid however can be inaccurate by introducing numerous artificial slip streams in evaluating the viscous-inviscid interaction from multiple intersecting shock waves. This issue and the high-order method development for the unstructured grid method remain as the mainstay for CFD research into the future.

A major pacing item for CFD adopting shock capturing techniques is evaluating discontinuous solution generated by shock waves and slip streams from shock interceptions. A breakthrough by Godunov demonstrates a multi-dimensional flow field that contains shock waves and contact surfaces can still be analyzed [16]. The discontinuities of the hyperbolic differential systems are treated as a piecewise continuous data distribution within a control volume and to be solved across the singular point as the Riemann problem. The underpinning principle is the monotonicity preserving property of the hyperbolic difference equation; namely, temporal increment/decrement of dependent variable is monotonic. Based on this property, Harten originates the total variation diminishing (TVD) scheme and spans off a huge amount of research on TVD schemes and a variety of flux limiters for analyzing piecewise discontinuous solutions for CFD [23].

From the physics viewpoint, the treatment of shock jump by flux splitting can be easily understood through the concept of zone of dependence for supersonic flows. By solving a set of Riemann problems over the entire computational domain according to their distinctive characteristics, this approach actually honors the physics of domain of influence. The directional wave propagation is constructed according to the phase velocity from the permissible database. In an outstanding work by Steger and Warming, it has shown a systematic relationship of the real eigenvalue and eigenvector for the split flux formulation. They also demonstrate the Euler equations, together with the equation of state for gas, possessing the homogeneous function of degree one property [24];

$$
\begin{aligned}
F_{i} & =\left(\partial F_{i} / \partial U\right) U=\left[A_{x}\right] U ; \quad G_{i}=\left(\partial G_{i} / \partial U\right) U=\left[A_{y}\right] U ; \\
H_{i} & =\left(\partial H_{i} / \partial U\right) U=\left[A_{z}\right] U
\end{aligned}
$$

where the $\partial F_{i} / \partial U, \partial G_{i} / \partial U$, and $\partial H_{i} / \partial U$ are the Jacobian matrices of the flux vector or the coefficient matrices. The governing equations in split flux vector form become;

$$
\partial U / \partial t+\partial\left[\left(F_{i}^{+}+F_{i}^{-}\right)+F_{v}\right] / \partial x+\partial\left[\left(G_{i}^{+}+G_{i}^{-}\right)+G_{v}\right] / \partial y+\partial\left[\left(H_{i}^{+}+H_{i}^{-}\right)+H_{v}\right] / \partial z=0
$$

The split flux vectors containing all inviscid terms are formed according to the signs of eigenvalue $\lambda$ of the coefficient matrices, $A_{x}, A_{y}$ and $A_{z}$; 


$$
\begin{aligned}
F_{i} & =F_{i}^{+}+F_{i}^{-}=\left(S_{x} \lambda_{x}^{+} S_{x}^{-1}+S_{x} \lambda_{x}^{-} S_{x}^{-1}\right) U \\
G_{i} & =G_{i}^{+}+G_{i}^{-}=\left(S_{y} \lambda_{y}^{+} S_{y}^{-1}+S_{y} \lambda_{y}^{-} S_{y}^{-1}\right) U \\
H_{i} & =H_{i}^{+}+H_{i}^{-}=\left(S_{z} \lambda_{z}^{+} S_{z}^{-1}+S_{z} \lambda_{z}^{-} S_{z}^{-1}\right) U
\end{aligned}
$$

In Eq. (8-c), the similarity and its inverse matrices for diagonalization of coefficient matrices are designated as $S_{x}$ and $S_{x}^{-1}$, the positive and negative eigenvalues are denoted as $\lambda_{x}^{+}$and $\lambda_{x}^{-}$. The solving procedure for the split equation is by applying one-side differencing approximation to achieve the approximate Riemann problem. The basic issue is that the split inviscid flux components are not differentiable at singular sonic points. This behavior is also the peculiarity of the approximate governing equations. The continuous viscous terms are solving simultaneously by spatially central scheme. An incisive summary for using the approximated Riemann approximations can be found from the work of Roe [25].

In spite of the rational treatments of discontinuous numerical solution, the undesirable artifact of numerical oscillation or the Gibbs phenomenon is always presented around a singular point. A series of excellent algorithms for maintaining computational stability and yielding sharp definition of a piecewise continuous numerical solution are the ENO and WENO (weighted ENO) schemes [26, 27]. The fifth-order WENO scheme is supported by an overall stencil of five points; the smoothness of a solution is measured by the sum of normalized squares of the scaled $\mathrm{L}_{2}$ norm for derivatives from the lower-order polynomials. The conditioned information is incorporated into the weights definition to improve the convergence at the critical points. It is revealed that the enhancement of the fifth-order scheme is derived from a large weight assigned to the discontinuous stencils, but not from their superior order of convergence at critical points.

From the lessons learned, the desired feature of a numerical scheme may be better selected from the optimization in the Fourier space rather than by focusing on the lowest possible truncation error. Along this line of reasoning, compact-difference scheme becomes a viable method to achieve high resolution. The basic algorithm is an implicit procedure for evaluating derivatives, which has a small stencil dimension and yet can maintain a lower level of dispersive and dissipative errors than the conventional numerical schemes. The basic formulation of compact-difference approximation is derived from the Hermite's generalization of the Taylor series [28]. The compact differencing formulations for evaluating the first-order and second-order derivative have been given by Lele [29] as;

$$
\begin{aligned}
& \beta(d U / d x)_{i-2}+\alpha(d U / d x)_{i-1}+(d U / d x)_{i}+\alpha(d U / d x)_{i+1}+\beta(d U / d x)_{i+2}= \\
& \quad c\left(U_{i+3}-U_{i-3}\right) / 6 \Delta x+b\left(U_{i+2}-U_{i-2}\right) / 4 \Delta x+a\left(U_{i+1}-U_{i-1}\right) / 2 \Delta x \\
& \beta\left(d^{2} U / d x^{2}\right)_{i-2}+\alpha\left(d^{2} U / d x^{2}\right)_{i-1}+\left(d^{2} U / d x^{2}\right)_{i}+\alpha\left(d^{2} U / d x^{2}\right)_{i+1}+\beta\left(d^{2} U / d x^{2}\right)_{i+2}= \\
& c\left(U_{i+3}-2 U_{i}+U_{i-3}\right) / 9 \Delta x^{2}+b\left(U_{i+2}-2 U_{i}+U_{i-2}\right) / 4 \Delta x^{2}+a\left(U_{i+1}-U_{i}+U_{i-1}\right) / \Delta x^{2}
\end{aligned}
$$

In practical applications, only the tridiagonal form is utilized and achieved by setting $\beta$ and $\mathrm{c}$ to zero to get the formulas relating the pertaining parameters.

$$
a=2 / 3(\alpha+2) ; \quad b=1 / 3(4 \alpha-1) ; c=0
$$

From Eq. (9-c), the fourth-order Pade formula is obtained by choosing the value of $\alpha$ $=1 / 4$, and the sixth-order derivatives are recoverable for $\alpha=1 / 3$ [29]. 
The formulas are three-point spatially central scheme and require attention on boundaries. Since the scheme is inapplicable on the immediately adjacent grid point next to a boundary, a transitional operator between the boundary and the interior domain is required. The transitional boundary scheme is not only required to transmit data from the boundary to the interior domain but also must preserve the stability and accuracy for the global solution. Although the high-resolution scheme is stable in the classic sense but the transition operator is one of the sources that contributes to spurious high-frequency oscillations known as time-delay instability. The time-instability is incurred by positive real eigenvalue components which dominate the numerical result.

A very effective remedy to control the time-delay instability has been demonstrated by using a low-pass filter [30]. The spectral function is a symmetric numerical filter that contains no imaginary part and has the low-pass amplitude response. In other words, the low-pass filter modifies only the amplitude but not the phase relation among all Fourier components. The tridiagonal spatial filter is given as

$$
\beta U_{i-1}+U_{i}+\beta U_{i+1}=\Sigma a_{n}\left(u_{i+n}+u_{i-n}\right) / 2 \quad n=0,1,2 \ldots N
$$

In Eq. (9-d), the variables $U$ and $u$ represent the filtered and raw data of the numerical solution. The free parameter is bounded by the values of $-0.5<\beta<0.5$. When the value approaches 0.5 , the coefficients of the symmetric filter can be obtained by expanding and matching the spectral function, $S F(\omega)=\Sigma a_{n} \cos (n \omega) /[1+2 \beta \cos (\omega)]$ by a Maclaurin series.

In Fig. 3, the accuracy and effectiveness of the 4th and 6th order compact-difference scheme with/without including some fine tuning is depicting to reduce significantly dispersive and isotropic errors in comparison with conventional numerical methods such as the 2nd order MUSCL and the simple 4th order explicit schemes. The detailed comparison on dispersive error is display in the wave numbers range of $\pi / 4<w<2 \pi$. The superior behavior of compact-difference schemes are noted, but the fine tuning may be counterproductive [31]. The reduction for isotropic error for multi-dimensional computation by compact-difference schemes is displaying in the wave numbers range from $\pi / 8$ to $3 \pi / 4$ in four quadrants. The failing of conventional methods are made in

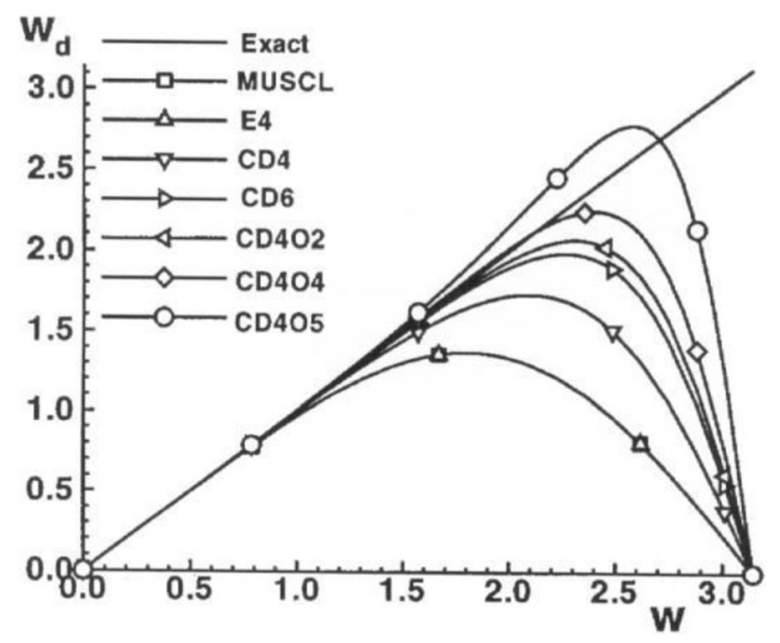

Fig. 3 Comparison of dispersion error of compact-difference scheme with others methods 
evident in Fig. 4; when wave number reaches the value of $\pi / 2$, which corresponds roughly to a grid-point density of 12 grid points per wavelength, and become unacceptable at the wave number of $3 \pi / 4$ for a sparser grid point density.

In most CD simulations, a high resolution solution is frequently needed in the high gradient regions like shock jump, media interface, and flame front. A local grid refinement approach appears to be very attractive; especially the grid refinement is independent from the global mesh system. The spectral-like polynomial grid refinement method introduced by Korpriva that meets the requirement, and the high resolution result is derived from the Gauss quadrature [32]. By this approach, there is no need to reconstruct the overall grid system, but by just increasing the degrees of polynomials within the refined grid block to capture the fine-structure features.

The local grid refinement numerical procedure is equally applicable to the temporal advancement of a time-dependent problem. In some cases, it may even be possible to examine a problem that has discontinuity between the integral intervals by relegating the singularity to the weighting function. A unique behavior of the recursive formula for derivative computation is that the result depends on all discretized points or the roots of the polynomial within the refined grid block. In fact, all high-resolution schemes striving to achieve a spectral-like accuracy are by employing all discretized points in an array to mimic the Gauss-quadrature formulation.

The spectral-like polynomial refinement for the entire computational domain is by dividing it into grid-refining blocks. The local high resolution solution is generated within each grid-refining block by the unequal-spacing roots from any of the classic orthogonal polynomials (Legendre, Laguerre, Chebyshev, Hermit, Gegenbauer, Jacobi, MeixnerPoluckzek). For discontinuity capturing, an artificial dissipative term may not be necessary within the subgrid domain for suppressing numerical oscillations, but just by reducing the grid-refining space dimension or by increasing the order of the polynomial. The local grid refinement approach has also been extended for solving the conservation laws on unstructured grids [33].

In the polynomial refinement approach for high local numerical resolution, the dependent variable, $U(x)$, is approximated by orthogonal polynomial through the Gauss

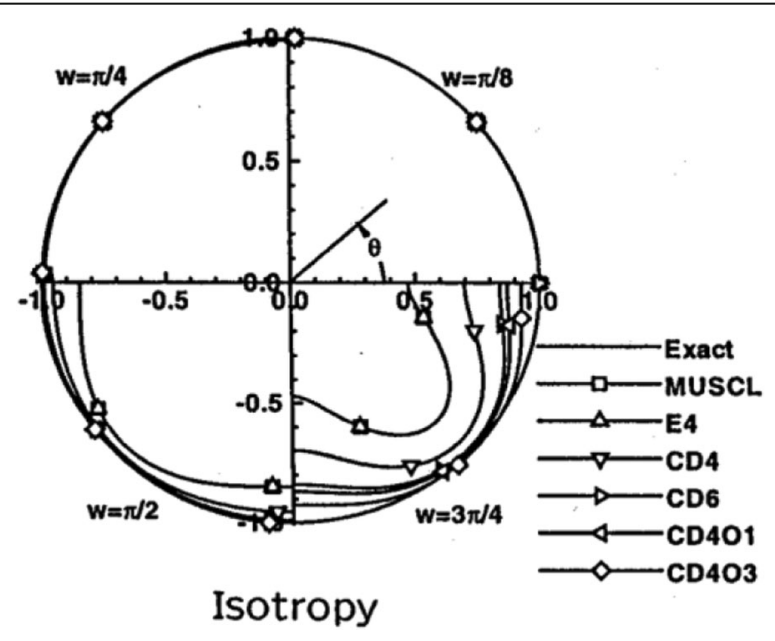

Fig. 4 Comparison of isotropic error of compact-difference scheme with others methods 
quadrature formula with a coordinate transformation of $x=[2 z-(a+b)] /(a+b)$ [34]. The integral limits of the grid-refining domain are designated by the values of $a$ and $b$ :

$$
\begin{aligned}
& \int_{a}^{b} U(\eta) d \eta=\frac{2}{n(n-1)}[U(a)+U(b)]+\sum_{i=1}^{n-1} L_{i} U_{i}(\eta)+R_{n} \\
& U(x)=P_{n}(x)+R_{n}(x)=\sum_{i=1}^{n} L_{i}(x) U\left(x_{i}\right)+\left[\prod_{i=1}^{n}\left(x-x_{i}\right)\right] \frac{U^{n+1}(\xi)}{(n+1) !}
\end{aligned}
$$

The numerical accuracy is uniquely determined by the chosen degrees of the ploynomial, as shown by the residue term $R n(x)$ of Eq. (10-b).

$$
L_{i}(x)=\prod_{\substack{j=0 \\ j \neq i}}^{n} \frac{\left(x-x_{j}\right)}{\left(x_{i}-x_{j}\right)}
$$

The weighted (Cardinal) function, $L_{i}(x)$, is generated by the Newton divided-difference formula for derivative calculation, and includes all unequal spaced roots of the orthogonal polynomial. This unique feature provides the spectral-like accuracy for the polynomial grid refinement method. In essence, the approximate dependent variable is defined by all grid points within the computational grid-refining block.

The derivative of any approximation functions with respect to either spatial or temporal independent variable can be computed by simply differentiating the Cardinal function $L_{i}(x)$ that has been generated by the Newton divided differnce formula; Eq. (10-c)

$$
\frac{d U\left(x_{i}\right)}{d x}=\sum_{n}\left[\frac{d L_{i}(x)}{d x}\right] U\left(x_{n}\right)=\sum_{i=1}^{l} \frac{\sum_{q=1, q \neq i}^{l}\left[\sum_{m=1, m \neq i, m \neq q}^{l}\left(x_{p}-x_{t}\right)\right]}{\prod_{m=1, m \neq i}^{l}\left(x_{i}-x_{m}\right)} U\left(x_{n}\right) . \quad(10-\mathrm{d})
$$

However, the differentiation procedure is best limited to calculate the first derivative from the approximate dependnet variable, because all othorgonal polynomials are generated by second-order hypogeometric differential equations. When attempt to use the approximate dependent variabe again to calculate the second derivative by the Cardinal function, the eigenvalue structure often leads to oscillatory behavior. For this reason, the second derivative shall be generated by the consecutive approximations using the calculated first derivatives as the input data.

Figure 5 presents the computation over a step function by the Gauss-Lobatto quadrature formula. A sudden drop of the dependent variable is prescribed near the coordinate origin from a value of 1500.0 at the coordinate origin to 300.0 into the computational domain. The discontinuity is resolved by reducing the quadrature interval by decreases the size of the grid-refining block. In turn, it redistributes numbers of points across the discontinuity. A decreasing integral interval from 0.02 to $0.000625 \mathrm{~cm}$ was performed to demonstrate the characteristic of the local grid refinement technique. In the numerical illustration, a 50-degree Legendre polynomial was used for all the calculations by the Gauss-Lobatto formulation. It is observed that by increasing the local grid refinement, the grid points are relocating along the vertical jump. 


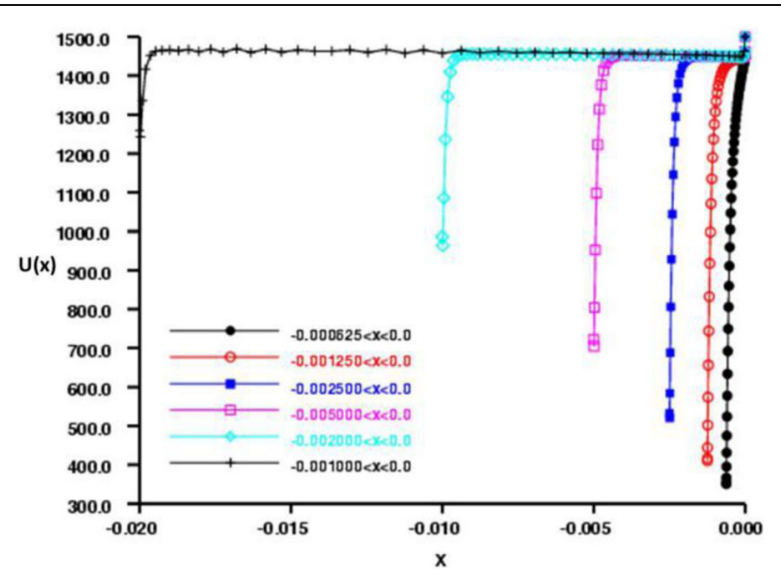

Fig. 5 Gauss-Lobatto quadrature across a finite Jump

A flame front in a bench mark case for hydrogen combustion experiment with shock acoustic wave has been conducted by the identical numerical algorithm with a 4th degree polynomial [35]. In Fig. 6, the superior and non-oscillatory numerical result for capturing a temperature spike across a hydrogen flame front is displayed by the Gauss quadrature algorithm in comparison with a 2nd order explicit finite-differencing method. The flame thickness is determined to be less than $0.5 \mathrm{~mm}$ to agree well with experimental data [36].

For finite-volume formulation, the basic approach is to generalize the Godunov's scheme and to extend it to an arbitrary order of accuracy for a hyperbolic differential equation. The perfect internal cancellation of flux vectors across control surface between elementary volumes known as the telescope property that makes the conservation of flux vector easily enforceable. Harten and his colleagues have shown by a cell-center formulation using reconstruction method to achieve a superior performance in the high wavenumber range with a lower truncation error [37]. In addition, the algorithm also avoids the Gibb's phenomenon at the discontinuity. The reconstruction algorithm is derived from an interpolation technique applying to a piecewise smoothed data. The basic formulation in the semi-discrete space combines the primitive functions, $u_{p}$ with multiple spatial cells and optimizes to

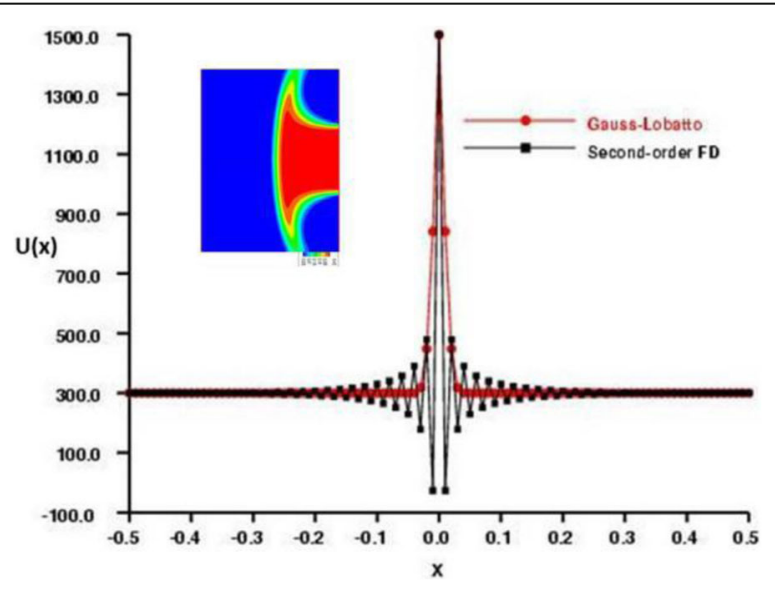

Fig. 6 Refined resolution over flame front by Guass-Lobatto quadrature 
minimizing dispersion and isotropic error. The flux reconstruction is defined by a primitive function as

$$
u_{p}=\int_{0}^{x} u d x
$$

By the second fundamental theorem of calculus, smooth solutions of the desired pointwise values are obtainable to the $\mathrm{n}^{\text {th }}$ order of accuracy. As we will see later, all the high-resolution algorithm development using polynomial approximation are derived from the reconstruction concept.

$$
u_{i+1 / 2}=d u_{p} / d x+O\left(\Delta x^{n}\right)
$$

For example, the smooth solution has been obtained by a 5-point compact stencil formulation,

$$
\alpha u_{i-1 / 2}+u_{i+1 / 2}+\alpha u_{i+3 / 2}=b\left(u_{p, i+5 / 2}-u_{p, i-3 / 2}\right) / 4 \Delta x+a\left(u_{p, i+3 / 2}-u_{p, i-1 / 2}\right) / 4 \Delta x
$$

The optimization is achievable by a spectral function with the modifying parameter of the compact-difference formula for an improved wideband wave characteristic in the high wave number range [31].

In the past decade, the Discontinuous Galerkin (DG) scheme [38] is probably the most investigated numerical algorithm. The original studies of the DG scheme in CFD is focused on solving the piecewise continuous hyperbolic partial differential equation or the Euler equations. The method have two outstanding features shared with both the finite-element and finite-volume methods: First, the high numerical accuracy of numerical result is derived from the high-order polynomials within an element or cell. Second, the propagation of data across a discontinuity is approximated by the Riemann problem.

From the finite-element view point, the formulation of the DG algorithm is developed by the weak formulation of the Euler equation multiplying with a weighting function $w$. The governing equation in vector flux form, Eq. (7-a) using the integral formulation can be expressed as;

$$
\int_{v}(\partial U / \partial t) w d v+\int_{s} F_{i} n_{i} w d s-\int_{v} F_{i}\left(\partial w / \partial x_{i}\right) d v=0
$$

where the symbol $n_{i}$ denotes the outward normal unit vector in the second integral over the element surface. In the semi-discrete form, the variable and weighting function are represented by the finite-element approximations with an analytic solution to appear as

$$
\partial / \partial t \int_{v} U w d v+\int_{s} F_{i}(U) n_{i} w d s-\int_{v} F_{i}(U)\left(\partial w / \partial x_{i}\right) d v=0
$$

Assume the approximate solution and the weighting function to be a piecewise approximate polynomial, then, 


$$
\begin{aligned}
& U(x, t)=\sum_{i=1}^{n} U_{i}(t) B_{i}^{p}(x) \\
& w(x)=\sum_{i=1}^{n} w_{i} B_{i}^{p}(x)
\end{aligned}
$$

Where $B_{i}^{p}$ is the basis function approximated by a polynomial of degree $p$. The finite-volume DG scheme is similar to the finite-element formulation. In fact, the first-order finite-element scheme is identical to the finite volume formulation but with a constant piecewise continuous polynomial [39]. In the above high resolution formulation, the temporal discretization is approximated by the explicit multi-stage TVD, Rung-Kutta scheme like all other early stage development of the DG for CFD applications.

The DG scheme offers distinctive advantages for high-performance CFD simulations; because it applies equally well to unstructured grid, as well as, multi-domains problem with local grid refinement. Again the individual cells or elements can be processed independently to minimize the data communication for highly scalable concurrent computations. This attractive feature has been identified as one the three crucial requirements for scalable parallel computations. The DG scheme has been extended to solve Navier-Stokes equation, with the implicit discretization by the upper-lower symmetric Gauss-Seidel (LU-SGS) algorithm [40]. Intensive research and comparative studies have been maintained for the DG applications to solve the Navier-Stokes equations in the areas of stagger grid (SG), spectral volume (SV), and spectral difference (SD) formulations. A wide range of improvements to computational efficiency and accuracy are made possible, because the basic DG scheme is based on polynomial approximation that can accommodate most adaptive strategies from unstructured grid to multi-gird block grid refinement techniques [41].

Another recent aerodynamic optimizations technique has shown impressive progresses for solving the RANS equations with the discrete adjoint approach by the Krylov algorithm [42]. The solving scheme is basically an iterative generalized minimal residue method (GMRES) by preconditioning the adjoint matrix according to the distribution of the eigenvalues and dimension. The unique features of its flexibility and enhanced iterative convergence for the optimizing aircraft design are recognized and will be sustained into the future.

\section{Landmark achievements}

The first coherent development for CFD was initiated in the Las Alamos National Laboratory (LANL) of United States in the middle 1940's for nuclear weapon development. Von Neumann and his colleagues have generated a substantial amount of fundamental knowledge for CFD through the path-finding research $[19,43]$. He developed the critical stability criterion for finite-difference approximation and addressed the issue for resolving hydrodynamic shock [44]. At the same time frame, Taylor independently completed his work for a spherical blast wave computation and led to the equivalence theory for hypersonic flow [45]. In few years later, Harlow developed the particle in cell (PIC) method based on a combination of Lagrangian-Eulerian description for fluid particle motion, and is still in use today for Magnetohydrodynamic research and rarefied gasdynamics [10]. All these accomplishments are substantiated by proof in order to illustrate the validity of the 
approximations and the numerical accuracy. The rigorous process actually established the standards for all future CFD algorithm and numerical procedure research.

Unfortunately, all valuable information was largely unknown to the aerospace community. The design and analysis tools in the aircraft industry prior to 1970's were mostly developed from linearized potential flow theory, method of characteristics, and boundarylayer theory. The shortcoming of these modeling and simulation tools becomes glaring when applying to high-speed aerospace vehicle design. It was realized that the computational capability by solving the compressible Navier-Stokes equation must be achieved for any further practical progress.

The vision of a systematic CFD development for aerospace community was crystalized by NASA Ames Research Center in the later 1960's, and their solution to limited computational resource distribution is the process of peer review and open debate [46]. The most important contributions beyond science and technology are the tradition of unselfish knowledge sharing, mutual support in the CFD community, and managing science and technology development by a clear distinction between these activities. A very large number of scientists, engineers, and scholars worldwide were participated in the basic research together. The successful CFD activities quickly expanded to all NASA science and technology centers, research laboratories of Department of Defense, aerospace industry. The knowledge transfer in that period actually reverses the traditional mode; the knowledge transition is actually from government agencies to academia and industry. The intensive CFD research activities have been sustained over more than three decades to become an unprecedented revolutionary discipline for aerospace science and engineering. The impact of CFD also expands beyond aerospace industry; the innovative and surged computational resource demand actually ignites the rapid growth of Silicon Valley.

In 1969, MacCormack developed an explicit numerical algorithm for solving compressible Navier-Stokes equations based on the classic Lax-Wendroff scheme [47]. His numerical method provides the tool for simulating and understanding the strong viscous-inviscid phenomena, meanwhile exposed the weakness of rudimentary turbulent closure models. A typical and classic computational simulation for a compression ramp with strong viscousinviscid interaction including boundary-layer separation is presented by Fig. 7. The numerical result by solving the Reynolds-averaged Navier-Stokes (RANS) equations is fully validated by experimental observation for the recirculating separated flow, and the coalescing compression waves upstream at flow separation and reattachment.

In 1977, a first three-dimensional hypersonic problem was also successfully simulated using the MacCormack's explicit algorithm [48]. The computational domain of a strong

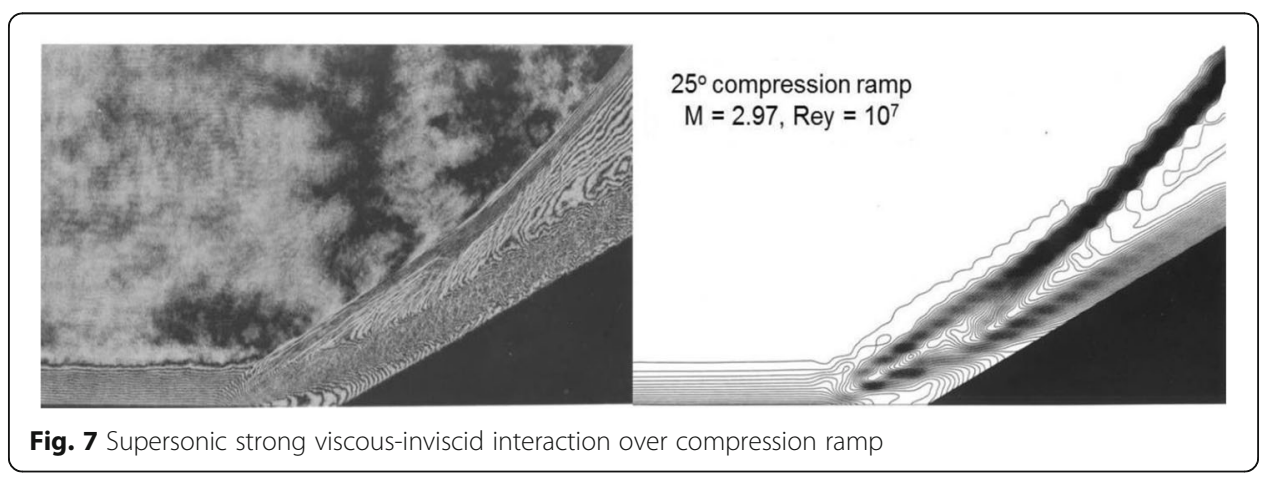


hypersonic shock-boundary-layer interaction was conducted in a frustum of a rectangular pyramid bounded by a wedge and a flat plate. A physically meaningful solution was generated on a very spare grid system, guided by the hypersonic equivalence principle in that the dominant flow perturbation occurs mostly in the cross-flow plane [49]. A triple-point shock structure in the corner flow region was captured by the numerical simulation, and correctly identified an intensive heat transfer spot adjacent to the corner juncture by a penetration inviscid stream beneath the triple-point. Although at that time numerical computations can only resolve the essential features of interacting flow fields, but CFD began to be recognized as a powerful tool for aerodynamic basic research. A sustained research effort for shock-shock and shock-boundary interactions has been maintained for the past fifty years, all these accomplishments have been summarized by Dolling [50].

Thompson and his colleagues first introduced the body orientated coordinate transformation technique which permitted CFD venturing into the reign of practical engineering application [9]. Their contributions have created a new CFD procedure to simulate fluid motion around practical and complex configurations. For structured grid computations, the grid generation technique evolved from the conformal mapping to transfinite method and by solving elliptic or hyperbolic partial differential equations. The generalized non-orthogonal three-dimensional coordinate transformation technique is crucial for applying CFD to aerospace engineering.

Another significant growth of CFD application is the coupling with chemically reactions, which also occurred in the early 1970's, by Spalding and his colleagues, and eventually integrated CFD to combustion and propulsive systems [51]. The interdisciplinary CFD technique has been refined by the significant progress in numerical algorithm and chemical kinetics modeling. The combustion in rocket, chemical laser system, and scramjet was accomplishments in the next decades [20]. These research efforts revealed a rapidly interdisciplinary advancement in CFD with nonequilibrium chemical kinetics.

In the same period, Jameson developed an explicit numerical procedure for transonic flows by solving the Euler equations [52]. He has made remarkable contributions in developing the shockless transonic wing, multigrid algorithm development, and several ingenious aerodynamic optimizing techniques. Meanwhile, major groups of two dimensional RANS simulations have also been devoted to investigate transonic airfoil included the near- and far-wake region. The theory of transonic small disturbance theory has been proved to be crucial in solving the flow past thin airfoils with imbedded shock waves. From the basic knowledge, the mixed elliptic-hyperbolic differential equation was solved by differencing formulas in the subsonic and supersonic regions to properly account for the local domain of dependence [53]. Equally important, outstanding CFD efforts have been exerted to explore previously unsolvable physics for airfoil buffet [54] and aileron buzz phenomena [55].

A group of implicit schemes for CFD was created to alleviate the conditional stability constraint inherent to explicit algorithms [56]. Another important step forward in CFD technology is the maturation of the finite-volume formulation. The finite-volume formulation of the macroscopic conservation law is intrinsic to the Eulerian frame of reference. This formulation is also less susceptible than the finite-difference approximation to the carbuncle anomalies at the axis of symmetry associated with the singular metric of coordinate [17]. However, the finite-volume method was not widely used until the 1980's. 
In the first decade of CFD development, the application to aerodynamics was limited mostly to canonical problems or aerodynamic shape that can be described by analytic means. For an example, the asymmetric vortical formation in the lee-side of a sharped-nose cylinder at a high angle of attack was illustrated and validated by experimental observations [57]. An even more fundamental challenge of examining boundary-layer stability was tackled by Fasel solving the Navier-Stokes equations [58].

In a short period of little less than a decade, aided by the rather crude and heuristic turbulence models, success simulations were achieved for aerodynamic phenomena over a wide range of Reynolds and Mach numbers. Equally important, CFD has contributed significant knowledge for flow separation and flows over arbitrary aerodynamic configuration; the knowledge gained is exemplified by the thorough understanding of separated flow topology and aerodynamic hysteresis of bifurcation [59]. The newly gained knowledge becomes invaluable for understand the intrigue shear flow patterns over most aerospace vehicle to improved aerodynamic performance.

In spite of all these convincing demonstrations, the objective of practical application of CFD for engineering design was yet to be achieved. Efforts to develop more efficient finite differencing schemes led to a group of implicit and hybrid procedures [20]. On the other hand, a class of computers designed for vector processing; the CRAY-1, STAR 100, and ILLIAC IV became available. At that period, the performance of a typical three-dimensional Navier-Stokes program on the CRAY-1 computer has achieved a data processing rate of 42.9 MFLOPS (million floating point operations per second) in the vector mode which was hundred times faster than the rate of a scalar computer. Rapid progress was also made for simulating airfoil and wing, wing-fuselage, afterbody, wake, combustion, propulsion system, and unsteady phenomena. Various simplifications to the Navier-Stokes equations were also developed by the parabolized and the thin-layer approximations for more efficient computational simulations [17].

The first ever CFD simulation of a complete aerospace vehicle configuration was accomplished in 1986 for X-24C-10D, which is a complete reentry vehicle with a blunt leading edge forebody, canopy, strake, fin, and wing [60]. A series of boost-glide vehicles were built for exploring the landing characteristics for low lift-drag ratios performance and unknowingly provided a design data base for the Space Shuttle. The numerical solution was generated at the Mach number of 5.95, a characteristic Reynolds number of $16.4 \times 10^{6} / \mathrm{m}$, and at a six-degrees-of-angle-of-attack to duplicate the experimental condition. The numerically predicted aerodynamic performance data for $C_{L}$ and $C_{D}$ are within $6 \%$ of the wind tunnel measuring error band. The side-by-side comparison of the surface shear flow pattern by numerical simulation and the surface oil flow is presented in Fig. 8 includes lines of scavenging and converging shear flow pattern on the vehicle surface. The central fin was found to be ineffective, because it is embedded deeply within the aerodynamic shadow, and was eliminated from future design. As a benchmark, the CFD technique was recognized as a critical technology for National Aero-space Plane (NASP) and Space Shuttle applications.

In the next five years, the CFD capability was rapidly developed to evaluating aerodynamic performance for aircrafts. The first aircraft simulation was focused on the F-16A fighter through a close collaboration between the Air Force Research Laboratory, NASA Ames Research Center, and General Dynamics. The CFD simulations were also applied to study the vortex breakdown and tail buffet phenomenon of the F-18 fighter. The modeling and simulation capability was also used to analyze the airborne laser turret, the structural 


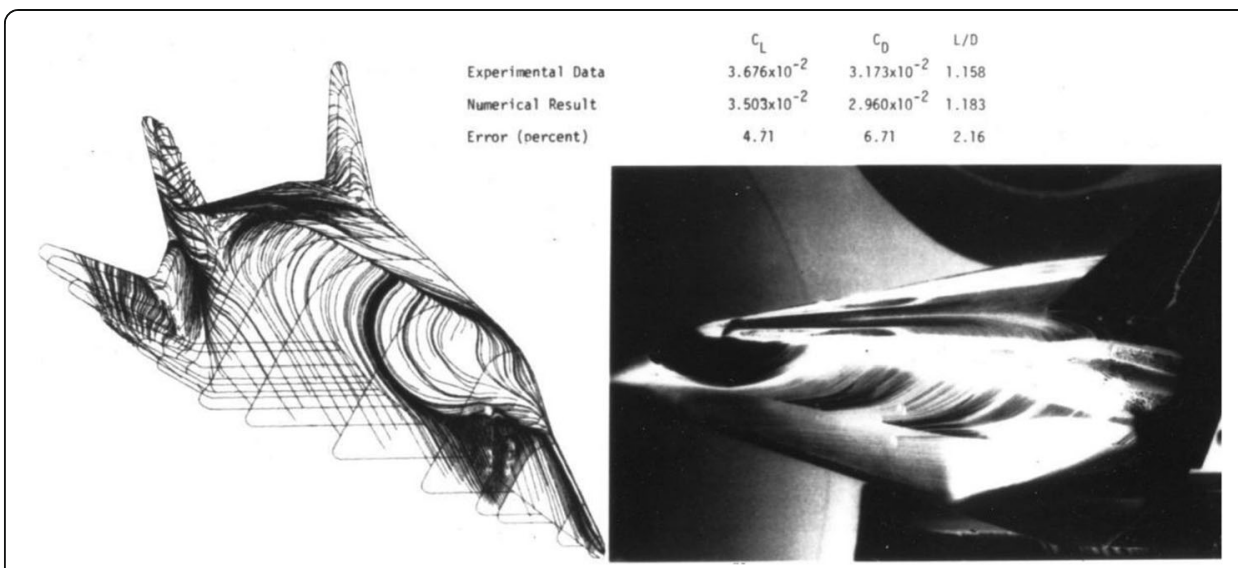

Fig. 8 Comparison of computational and experimental observation of X-24c reentry vehicle

response to a flexible vertical tail of the F-15, and for many advanced fighter aircraft designs. Equal numbers of application have focused on high-lift civilian transport design and analysis; such as the Airbus 380, Boeing 737, and 777-200 [20].

The CFD applications in the 1990s' were dominated by NASP and Space Shuttle operations. The NASP Program initiated from 1986 through to 1994 was the principal motivator for progresses in CFD. Nearly all sectors of the US national laboratories, NASA Centers, aerospace industry, and more than fourteen major universities are actively participated in this program [61]. The NASP design was and still is a quantum leap from the traditional approaches to aircraft and space vehicle design. The demonstration aircraft, X-30, was to operate through the atmosphere from subsonic to orbital velocities at a Mach number exceeding 25 . The challenge in developing the high-temperature material for vehicle fabrication was one of the major issues. The design and analysis of X-30 must integrate multiple engineering disciplines consisting of aerodynamics, propulsion, structure, and flight control. The capability for airframe design and flight control/stability was not an overreach, but the required propulsion systems based on subsonic and supersonic ramjet combustion faced a formidable challenge [62].

The CFD application to the Space Shuttle accelerated after the Challenger (STS51-L) accident in 1986 [63]. A higher accuracy requirement was imposed to predict the orbiter wing root shear to within $5 \%$ of the maximum structural capability of the wing. The multiple-zonal grid consists 111 grid blocks with a 16 million grid point system, and the surface resolution on average was about $10 \mathrm{~cm}[64,65]$. Figure 9 depicting all the possible perturbations to flowfield by fine-scale structures in the grid generation process on the external tank. The full-scale, high fidelity grid generation of the Space Shuttle launch vehicle with all fine-scale details was using the ICEM/CFD $\mathrm{CAD}$ and gridding software. The earlier numerical solutions for the vehicle were obtained using the chimera domain decomposition technique, and then logically transitioned into the unstructured grid method to describe the complex and multi-component configurations. The simulated Space Shuttle configuration consists of the orbiter, external tanks, plus two solid rocket boosters, and the complex multiple shock-on-shock interactions are capturing by the solving procedure. The CFD simulation for space shuttle in launching configuration represents the state-of-the-art at that period and is displaying by Fig. 10. 


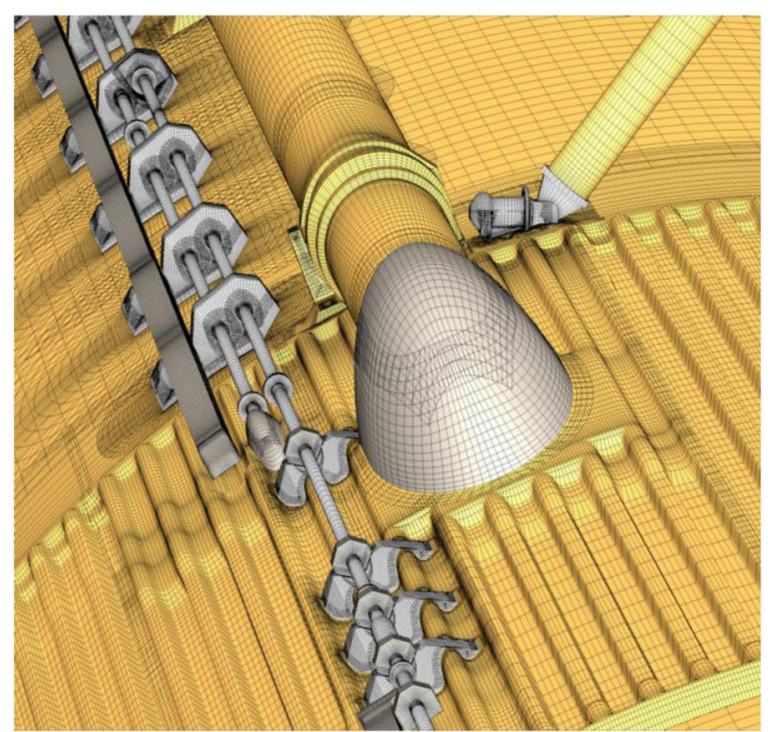

Fig. 9 Required fine-scale geometric details space shuttle external tank [64]

The increasing complexity in physics and managing a huge amount numerical data for practical applications put a tremendous pressure on improving computational efficiency for the even higher data processing rates and large memory storage. The parallel or concurrent computing with shared or distributed memory by a cluster of multiple computers or reduced instruction set computers (RICS) was the solution offered by the computing technology community. At the early stage of concurrent multi-computers development, there were a group of different shared-and distributive- memory computers (Cray T3E, IBM SP, SGI Origin 2000, N-cube and others). Therefore the strategy of porting the most

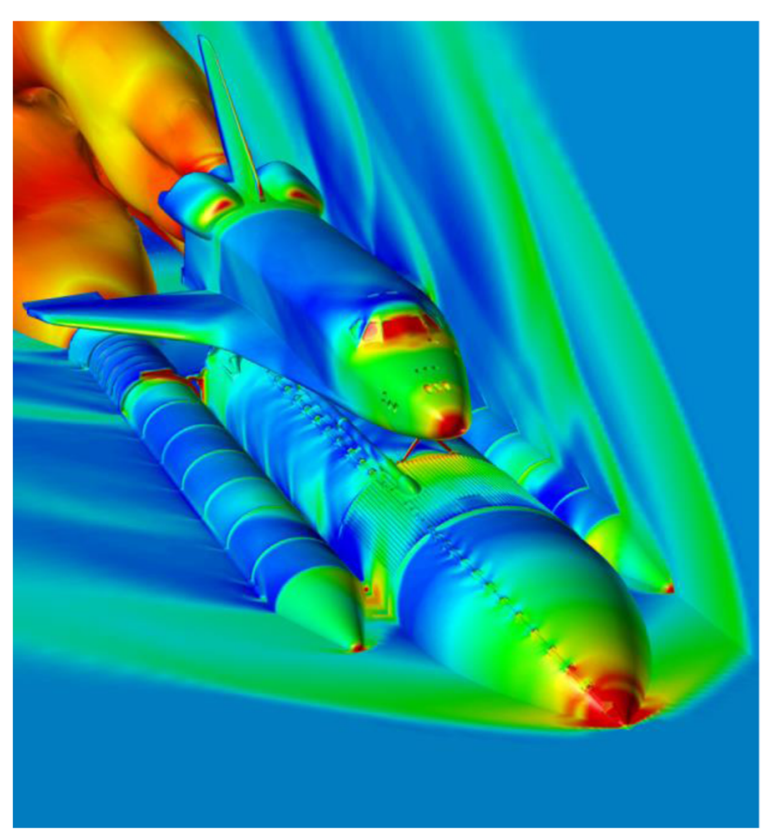

Fig. 10 CFD simulation of space shuttle launching configuration [96] 
sequential CFD computer codes adopted the domain decomposition approach because it was the most flexible and efficient data partition. The message passing interface (MPI) becomes the mainstay of concurrent computing paradigm [66]. In the early 2000's, the data processing rate had reached a value of one GFLOPS (Giga floating point operation per second, $10^{9}$ FLOPS) and the size of distributed memory has approached a terabyte. From basic research result, the load balancing, communication minimization among processors, and efficient cash memory utilization were found to be paramount for high parallel numerical simulation efficiency [67]. The versatility of unstructured Navier-Stokes/Euler solvers has been demonstrated for aircraft design. The most noticeable accomplishments were the computational simulations for Boeing 737, 777-200, and Airbus 330. A dream that one can evaluate the aerodynamic performance of any complete aircraft in a few weeks has been realized.

However, the leading stumbling block in fluid dynamics is still the turbulent flow, which is strictly a chain of events from the unsteady, three-dimensional, nonlinear vortices interaction with a wide range of length scales and frequency spectra. The dimensions of vortices vary from the Kolmogorov inner scales to large-scale vorticities comparable to the entire flow field [68]. The finest length scale of turbulence is defined as $\left(v^{3} / \varepsilon\right)^{1 / 4}$ which involves the kinematic viscosity $v$ and turbulent kinetic energy dissipation rate $\varepsilon$. The time and velocity scales also knew as $(v / \varepsilon)^{1 / 2}$ and $(v \varepsilon)^{1 / 4}$ respectively. At the atmosphere condition, the Kolmogorov scale has a dimension around ten millimeters. For simulating aircraft in flight conditions by directly solving the Navier-Stokes equations (DNS) requires a mesh system of more than quintillions of points and a data processing rate of hundreds of petaflops $\left(10^{18}\right.$ floating point operations per second) [69]. An engineering alternative becomes necessary.

According to our physical understanding of wall-bounded turbulent flow, the larger eddies extract energy from the main flow and its structures are highly anisotropic. On the other extreme, the small eddies dissipate energy, tend toward isotropy and are nearly universal in character. In Fig. 11, the streamwise turbulent energy spectra for various type of wall-bound flows is assembled [46]. It is clearly demonstrated that near the wall or in the inner region of turbulent shear layers, the spectra of the small energy-dissipating eddies are clearly universal - it is independent of both Reynolds number and type of flows. This is the guiding principle for subgrid scale modeling for large eddy simulation (LES) [70].

For LES formulation, the compressible Navier-Stokes equations are decomposed by the scales of temporal and spatial independent variables. The large-scale turbulence is resolved by solving the time-averaged Navier-Stokes equations over the large-scale motion, and the small-scale turbulence is approximated by some dynamic subgrid models [71]. The temporal scales separation for LES formulation is achieved by the Reynolds-average, but truly is the mass- or Favre-average. The temporal ensemble is obtained by some predetermined but arbitrary characteristic time scales;

$$
\tilde{f}=\frac{\int \rho f d t}{\int \rho d t}
$$

and the small spatial scale fluctuations are eliminated by a low-pass filter $G(x-x)$;

$$
\bar{f}=\int G\left(x-x^{\prime}\right) f^{\prime}\left(x^{\prime}\right) d x^{\prime}
$$

After a rather arbitrary separation of the large and small scale eddies, the governing equation of LES acquires the following form; 


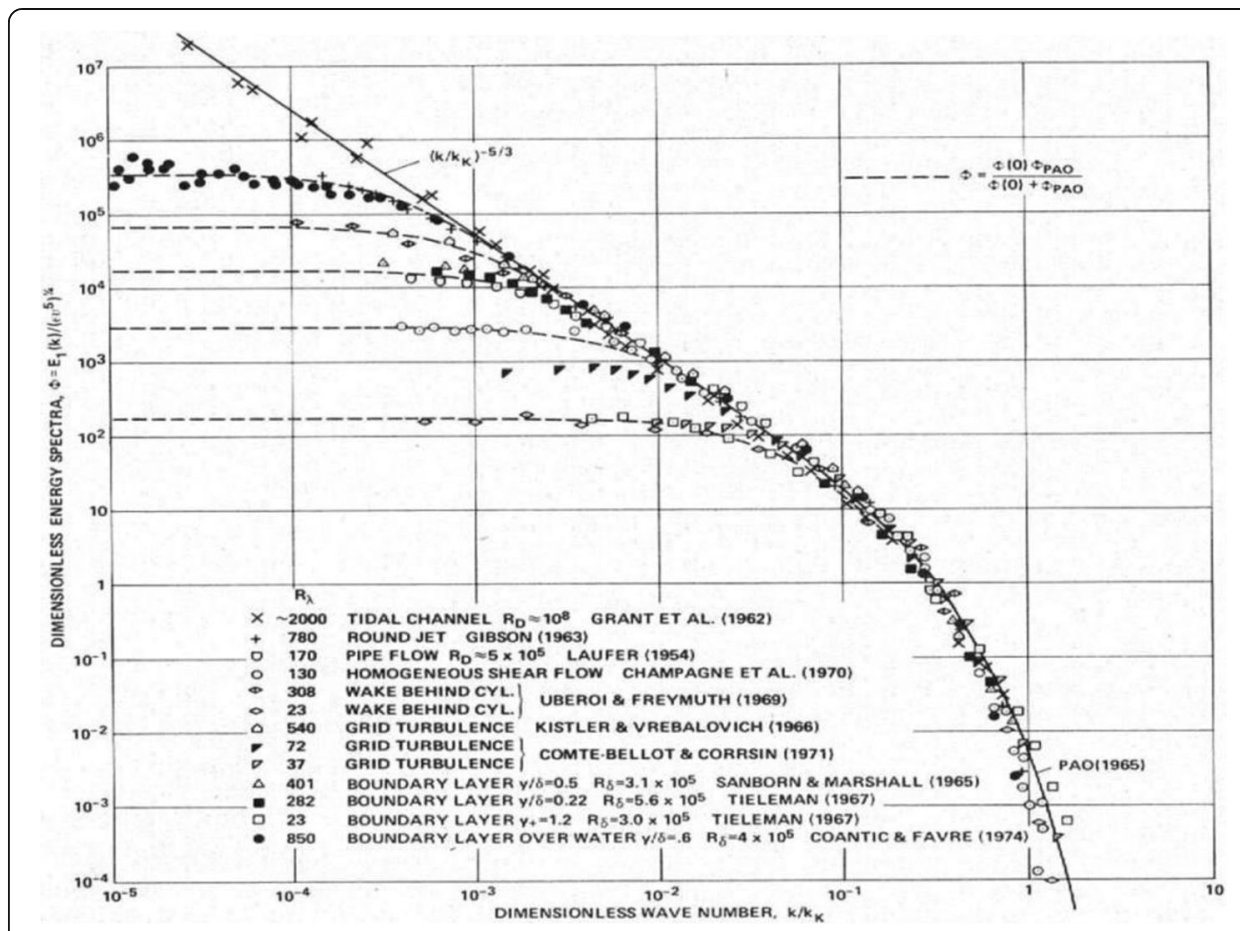

Fig. 11 Streamwise turbulent energy spectra for various turbulent flows [46]

$$
\begin{aligned}
& \partial \bar{\rho} / \partial t+\nabla \cdot\left(\overline{\rho u_{i}}\right)=0 \\
& \partial \overline{\rho u_{i}} / \partial t+\nabla \cdot\left(\bar{\rho} \overline{u_{i} u_{j}}\right)=(1 / \operatorname{Re}) \partial \bar{\tau}_{i, j} / \partial x_{i}-\partial \tau_{i, j} / \partial x_{j} \\
& \partial \overline{\rho e} / \partial t+\nabla \cdot\left(\bar{\rho} \overline{u_{i} e}+\overline{u_{i} \cdot \tau_{i, j}}-\bar{q}_{i}+q_{i}\right]=0
\end{aligned}
$$

The closure of the above equation systems are provided by the subgrid scale stress (SGS) tensor $\tau_{i, j}=\bar{\rho}\left(\overline{u_{i} u_{j}}-\bar{u}_{i} \bar{u}_{j}\right)$ and heat flux $q_{i}=\overline{e u_{i}}-\bar{e} \bar{u}_{i}$. A range of the SGS models have been developed and loosely grouped as the eddy viscosity, dynamic eddy viscosity, and similarity models [72]. The numerical results by LES when comparing with experimental observations have exhibited close correspondence in distributions of standard deviation, skewness, kurtosis, and intermittency of turbulent dynamics.

The rapid maturation of CFD for aerospace applications and supporting by parallel computational become directly usable for engineering. From the lessons learned in the aerospace vehicle design, the integration of aerodynamics, propulsion, structural dynamics, and flight control shall be implemented at the preliminary design stage to minimize the inefficient iterative cycle. The data base for the final design has also gradually shifted from experimental to computational simulations and finally verified by flight test. The computational simulation, in spite of the obvious shortcomings in predicting laminar-turbulent flow transition and turbulent, is very reliable for interpreting the flow behavior between two adjacent states. This feature has been adopted for comparative study to determine the final vehicle configuration. It also realized for high-speed flight in a high enthalpy environment, the governing equations derived from the gas kinetic theory are inadequate for modeling flow medium that are significantly affecting by internal degrees of excitations from vibration, disassociation to ionization. These phenomena stand out for hypersonic flight, earth reentry 
from space exploration, and applying the electromagnetic force/energy for flow control and performance enhancement.

For high-speed aerodynamics simulation, the nonequilibrium thermodynamic and chemical reaction within the enveloping bow shock must be examined. The temperature of the compressed air within the hypersonic shock layer routinely exceeds ten thousand degree Kelvin, where the nonequilibrium thermodynamics and chemical kinetics phenomena dominated. The physical fidelity requirement thrusts CFD into the modeling for atomic/molecular internal degrees of excitation by quantum physics. The works of Park [73], Candler and MacCormack [74], and Josyula and Shang [75] exemplified this path-finding research and opened a new research arena for CFD. Meanwhile, the needs of stealth technology and embedded antenna design also inspired the knowledge transfer from CFD to computational electromagnetics (CEM) in the time domain. This need was intended to augment the radar cross section (RCS) prediction from the ray-tracing technique using optical physics to CEM in the time and frequency domain. The interaction between CFD, CEM, and CMA (computational magneto-aerodynamics) needs to sustain into the future.

The interdisciplinary CFD (ICFD) governing equations are shared for analyzing the hypersonic flows and the multi-fluid and multi-temperature plasma. Under the low magnetic Reynolds number condition $\left(R_{m}=\sigma \mu u_{\infty} L, \sigma\right.$ denotes the electric conductivity of the medium) and by restricting the plasma frequency within the microwave spectrum, the Faraday induction law can be decoupled from Maxwell's equations. This simplification focuses the study of magneto-aerodynamic interaction to fluid motion rather than on electromagnetic waves propagation. By this formulation, the electrostatic force, the Lorentz acceleration, and the Joule heating appear as the source terms in the ICFD equations. The essential physics of aerodynamics-electromagnetics interaction for ionized gas can be effectively approximated by a simplified interdisciplinary governing equations system [35]:

$$
\begin{aligned}
& \frac{\partial \rho_{i}}{\partial t}+\nabla \cdot\left[\rho_{i}\left(u+u_{i}\right)\right]=\frac{d w_{i}}{d t}, \\
& \frac{\partial \rho u}{\partial t}+\nabla \cdot(\rho u u+p-\tau)=\rho_{e} E+(J \times B), \\
& \frac{\partial \rho e}{\partial t}+\nabla \cdot\left[\rho e u-\kappa \nabla T+\sum \rho_{i} u_{i} h_{i}+q_{r a d}+u \cdot p+u \cdot \tau\right]+Q_{v t}-Q_{e t}=E \cdot J .
\end{aligned}
$$

The vibrational energy conservation equations for polyatomic molecular species are

$$
\left.\frac{\partial \rho_{i} e_{i V}}{\partial t}+\nabla \cdot\left[\rho_{i}\left(u+u_{i}\right) e_{i V}+q_{i V}\right)\right]=e_{i V} \frac{d w_{i}}{d t}+Q_{V, \Sigma}
$$

The electronic energy conservation equation has been traditionally given as

$$
\begin{gathered}
\left.\frac{\partial \rho_{i} e_{e}}{\partial t}+\nabla \cdot\left[\rho_{i}\left(u+u_{i}\right) e_{e}+u \cdot p_{e}+q_{e}\right)\right]=e_{e} \frac{d w_{i}}{d t}+E \cdot J \\
+\left[\rho_{e} E+(J \times B)\right] \cdot\left(u+u_{i}\right)+Q_{e, \Sigma}
\end{gathered}
$$

The definition of the internal energy is now generalized to appear as, 


$$
\rho e=\sum_{i \neq e} \rho_{i}\left(c_{V, i} T+\frac{u \cdot u}{2}\right)+\sum_{i \neq e} \rho_{e} e_{V, i}+\sum_{i \neq e} \rho_{i} h_{i}^{o}+\rho_{e}\left(c_{V, e} T_{e}+\frac{u_{e} \cdot u_{e}}{2}\right) \quad(14-\mathrm{f})
$$

where $h_{i}^{o}$ is the standard heat of formation for all reacting species.

The production and depletion rates for gas species of different energy states $\left(d w_{i} / d t\right)$ in the continuity equation, Eq. (14-a), are calculated by the law of mass action for chemical kinetics. The diffusion velocity, $u_{i}$ of different species is determined from the gas kinetic theory. In Eq. (14-c) through (14-e), the energy cascading from the translation, vibration and electron excitations of gas species, $Q_{v t}, Q_{e t}, Q_{v, \Sigma}$ and $Q_{e, \Sigma}$ by quantum jumps are modeling either by the principal of detail balancing or by empirical formulations. Again in the governing equations, (14-b), (14-c), and (14-f), the electrostatic force $\rho_{e} E$, Lorentz acceleration, $J \times B$ and Joule heating $E \cdot J$ represent the coupling between fluid motion and electromagnetic force/energy. The electric and magnetic field intensities $E$ and $B$ are the sum of the externally applied and the induced field intensities. In the absence of an externally applied magnetic field, the charged particles are accelerated by the electrostatic force alone due to charge separation in free space. These numerical simulation capabilities become the backbone of engineering design and analysis for hypersonic flight, reentry thermal protection [76, 77]. Computational simulations have been successfully applied to all space vehicle programs from the first FIRE reentry probe prior to the Apollo mission, the radio attenuation measurement probe RAM-C, Orion, to Stardust for earth reentry. Numerical simulation also carried out for entry into other planets such as the Pioneer to Venus, Galileo probe to Jupiter, as well as Neptune and Titan.

Among all reentry investigations, the Stardust reentry probe exemplifies the shape of reentry vehicle and enters the earth atmosphere with the highest speed than all other man-made objects. A common feature for all reentry vehicles has a blunt forebody to reduce the maximum stagnation point heat transfer rate. The forebody of Stardust consists of a 60-degree one-half angle spherical cone with a nose radius of $0.229 \mathrm{~m}$. The afterbody is a truncated 30-degree cone with a base radius of $0.406 \mathrm{~m}$. The corner radius at the juncture of the forebody and afterbody is merely $0.02 \mathrm{~m}$. The flowfield structures by velocity traces over the Stardust probe are depicted in Fig. 12; the probe reenters at an angle of attack of eight degrees and after $54 \mathrm{~s}$ elapsed from a designated reentry altitude. At this point in time, the probe is still traveling at the speeds of $11.37 \mathrm{~km} / \mathrm{s}$. The flow topology is shared by all the reentry vehicles; they all have a strong bow enveloping shockwave to contain a relatively thin shock later following by a rapid expansions between forebody and the afterbody, then emerging into the wake region. In the base region, the flow is separated from the vehicle downstream at corner region of the base to form recirculating flow with the lowest heat transfer rate. All these dominant flow topologies have been clearly captured and displayed by the computational simulations [78].

The RAM-C-II probe generated the most valuable verification flight data for the physical-based modeling with nonequilibrium chemical reaction in the shock layer, including the ionized gas spices. The probe consists of a hemispherical-nosed cone with a cone angle of 18 degrees and an overall length of $1.3 \mathrm{~m}$. The probe collects the ionized air data by a reflectometer and at a single double check point by an electrostatic device. The comparison between data and computational results for electron density is reasonable and reveals the electron density in the reentry environment have reached a nearly equilibrium state at the later stage of reentry (Alt $=61 \mathrm{~km}, \mathrm{M}=23.9$, and $\left.\mathrm{Re}=1.95 \times 10^{4}\right)$. During all the 


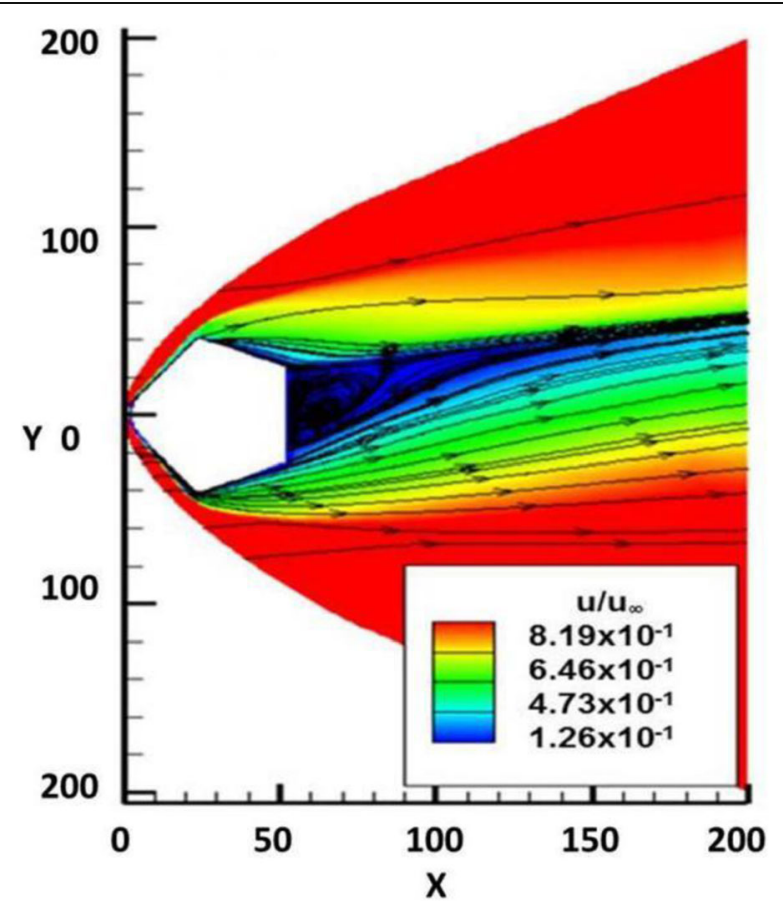

Fig. 12 Velocity traces of Stardust probe at reentry speed of $11.37 \mathrm{~km} / \mathrm{s}$

stages of reentry, the depletion of molecular oxygen and redistribution of nitro oxide in the stagnation region stands out during the earth reentry. All the translational, vibrational, and electronic temperatures (by assuming to have Maxwell distributions) attain the equilibrated condition approaching the stagnation point. The calculated species concentrations; $\mathrm{O}_{2}, \mathrm{~N}_{2}$, $\mathrm{NO}, \mathrm{N}$ and $\mathrm{O}$, as well as, the ionized components $\mathrm{N}^{+}, \mathrm{O}^{+}$, electron $\mathrm{E}^{-}, \mathrm{N}_{2}^{+}, \mathrm{O}_{2}^{+}$, and $\mathrm{NO}+$, along the stagnation streamline are displayed in Fig. 13. The agreement among computational simulations and flight data confirms that physical-based approximation to quantum mechanics is acceptable for engineering purpose, but does not necessarily represent the actual physics involves quantum jumps.

In the aspect of ICFD evolution, technique transition to computational electro-aero dynamics (CMA) has enhanced aerospace vehicle aerodynamic performance. The ion engine is one of the genuine successful CMA applications to aerospace engineering. Since 1998 the gridded electrostatic ion thrusters and the Hall effects thrusters have routinely been used for geosynchronous satellites and space stations orbital position keeping. Both ion engines operate in a rarefied gas environment, and the maximum electron/ion number density is around $10^{13} / \mathrm{cm}^{3}\left(10^{19} / \mathrm{m}^{3}\right)$ and each charged particle possesses an energy level over $3 \mathrm{eV}\left(1.6022 \times 10^{-12} \mathrm{erg}\right)$. Therefore, computational simulations must be conducting by either the PIC or PIC-DSMC methods. Although the thrust from ion engines is generally limited form hundreds of mille-Newton to a few Newton, and the output power is in the order of tens of $\mathrm{kW}$, but the life cycle of ion engines exceeds thousands of hours for deep-space exploration missions. The unique features of ion thruster are the high exhaust ion velocity which can be as high as $210.0 \mathrm{~km} / \mathrm{s}$ by an impulse magneto-plasma thruster, and a high specific impulse from 1700 to $12,000 \mathrm{~s}$. Innovation is still continuing for the development of the magneto-plasma-dynamic thruster. 


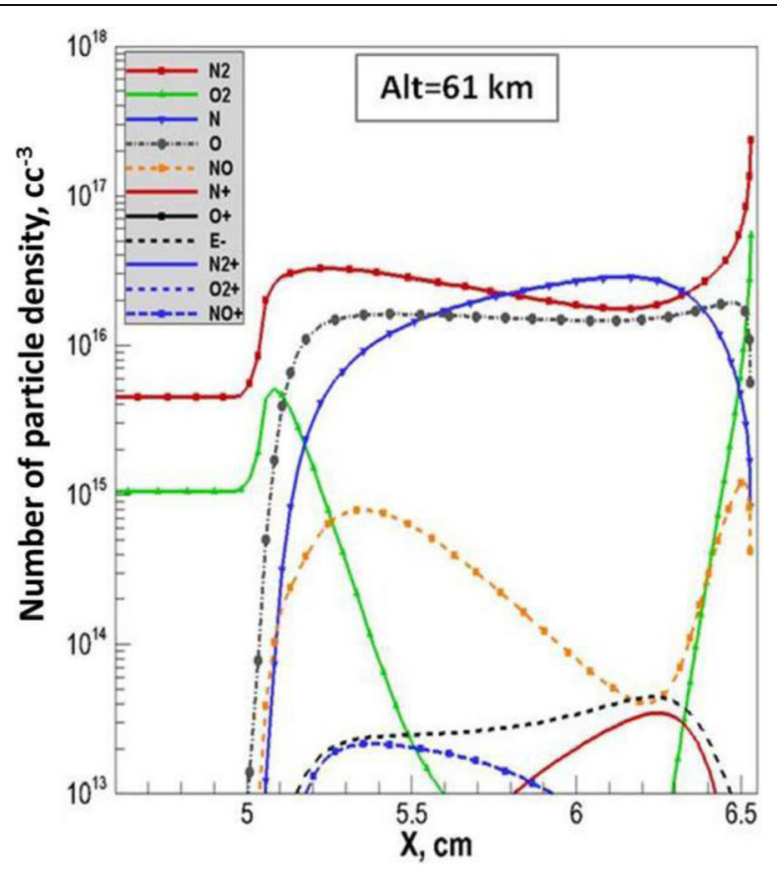

Fig. 13 Species concentration along stagnation streamline of RAM-C-II probe

Adopting plasma actuator for flow control for aerodynamic applications is very attractive, because the process does not require any movable components or servo mechanisms. The electrically conducting medium imparts additional electromagnetic force and energy onto the flow field by Joule heating, electrostatic and Lorenz acceleration [35]. The flow control based on Joule heating is through suddenly growth of the boundary-layer displacement by a localized cathode heating, which is effective for hypersonic flows at the outer atmosphere. On the other hand, the periodic electrostatic force across electrodes with an AC electric current in microwave frequency by the dielectric barrier discharge (DBD) is the most effective for flow control by the momentum transfer via collisions between ions with neutral particles. An induced jet-like jet stream over the overlapping electrodes can move at a velocity up to $10 \mathrm{~m} / \mathrm{s}$, but ceases beyond the thin cathode layer [79]. For these reasons, in a weakly ionized air, the added electromagnetic force and energy for flow control are presented only in the perturbation level to the main flow, therefore needs to amplify by aerodynamic viscous-inviscid interaction or by a strong externally applied magnetic field to be effective. The plasma flow control mechanisms, without a high-degree of ionization or a strong externally applied magnetic field, are applicable only for a special purpose implementation. However, the most recent research using plasma for ignition and maintaining combustion stability may result in unfathomable scientific innovations [80].

The technique transition from CFD to CEM is rested on the numerical algorithm for solving the hyperbolic differential equations. In specific, the characteristic-based formulation, the perfectly matched layer technique, and the dispersion-relationship-preserving scheme have alleviated one the two fundamental limitations for imposing far-field boundary condition for solving the Maxwell equations in the time domain. CEM has demonstrated fully for electromagnetic wave scattering prediction and antenna design up to the resonance frequency domain [35]. The second limitation imposed by the numerical resolution requirement for CEM is defined by the well-known Enquist limitation - At least two grid points 
per wavelength are required to resolve wave dynamics. For a millimeter microwave that must propagate over a hundred-kilometer distance, the needed grid-point number for a satisfactory numerical resolution along the beam path is astronomical. To date, the parallel computation capability has permitted the CEM venturing into optical frequency domain by an optical physics approximation through the ray-tracing method. The maturation of high-performance supercomputing technology with the data processing rate approaching the Exa floating operation points per second (EFLOPs, $10^{18}$ FLOPS) definitely will remove the last hurdle of CEM for practical aerospace applications.

In the early 2000s, the Micro aerial vehicle (MAV) and unmanned aerial vehicle (UAV) are another application of ICFD to aerospace engineering and become an interesting development in fluid dynamics [81]. It highlights the dynamic lift generation by vortex formation at extremely low Reynolds number condition for MAV. In the case for mimicking the bio-inspired design, the coupling of aeroelasticity and advanced flight control algorithm with fluid dynamics for reliable flight is essential [82]. On the other hand, the UAV operation requires precision guidance to long-endurance autonomous flight for extremely maneuverable flight vehicle. The broadened CFD applications accelerate the ICFD development into increasing complex scientific disciplines and demanding new numerical algorithm development on moving frame of references. The basic issues of hydrodynamic instability, laminar-turbulent transition, and turbulence emerge repetitively as the most sever challenge to computational simulations.

\section{New Frontiers}

From the brief reviews of the CFD history, it becomes clear that the formidable challenges before us are to resolve the least understood fluid dynamics phenomena and to expand the governing equations of CFD beyond the classic kinetic theory of gas. For the former, the issues of fluid dynamics bifurcations ranging from hysteresis, vortex breakdown, laminar-turbulence transition, to turbulence are easily identified. For the latter, the simplified elastic collisions between gas particles need to be replaced by the inelastic collision including the internal degrees of freedom for gas molecular and atomic excitations in high-speed, high-temperature flight environments.

Understanding fluid dynamic bifurcations by solving directly the Navier-Stokes equations is not necessarily a brute force effort but must be approach by a better understanding of the physics. For example; the vortical breakdown is trackable by the formation of the stagnation point in free space for a vortical motion [83]. The hysteresis of fluid motion is exclusively associated with a delay consequence of an initial condition. The laminarturbulent transition is arisen from hydrodynamic instability, environmental disturbances or receptivity, and the distribution of intermittency [84]. To analyze the most difficult turbulence is best by understand the phenomena of simultaneous interactions of large- and small-scale eddies in time and space with the ever present hairpin vortices that travel in packets over fluid-solid interface [68]. It is realized that there is a common dominator for better understanding of all these largely unknown phenomena, which is to resolve the fine temporal and length scales structures; either for turbulence, electromagnetic wave propagation, or nonequilibrium quantum chemical-physics. The crucial numerical resolution requirements for resolving these physics-based computational simulations are begin to be met by the high-performance supercomputers. 
Computational technology outgrows the Moore's and Amdahl's arguments. On June 2016, the Sunway Taihulight supercomputer clocked a data processing rate of 93.01 PFLOPS, running the LINPACK benchmark, and with a mindboggling memory capacity of $1.32 \mathrm{PiB}$ ( $2^{50}$ bytes). Exact two years later, the fastest benchmark data processing rate was claimed by the Summit supercomputer at 122.3 PFLOPS. It is anticipated that the data processing rate of supercomputer will attain a value over EFLOPS (1000 PFLOPS) within the next few years. The massive parallel computing technology has opened avenues for basic research and created new scientific frontiers in quantum physics, molecular dynamics, climate, and artificial intelligence research. In Fig. 14, the CFD requirements for direct simulating turbulence and nonequilibrium quantum chemical-physics are mapping onto the capability of supercomputers of today. The match of supply and demand shows the time is here for direct and real-time numerical investigations.

Based on our basic understanding of turbulence, which is consist of chaotic vortices interactions, the formulation of Navier-Stokes equations in vorticity and velocity is direct and more advantageous than the traditional conservative variables for study turbulence. The vorticity-velocity formulation of the three-dimensional, compressible Navier-Stokes equations by combining the continuity, momentum equations, and energy equation; Eq. (5-a) through (5-c) yield;

$$
\begin{array}{ll}
\partial \Omega / \partial t+\Omega(\nabla \cdot u)+(u \cdot \nabla) \Omega-(\Omega \cdot \nabla) u-\nabla[p-\lambda(\nabla \cdot u)] \times \nabla(1 / \rho) & (15-\mathrm{a}) \\
-\nabla \times[(\nabla \mu / \rho) \cdot \operatorname{def}(u)]-2 \nabla v \times[\nabla(\nabla \cdot u)]+\nabla v \times(\nabla \times \Omega)-v \nabla^{2} \Omega=0 & \\
\rho \partial\left(c_{v} T+u \cdot u / 2\right) / \partial t+\rho u \cdot \nabla\left(c_{v} T+u \cdot u / 2\right)+\nabla \cdot q+\nabla p \cdot u-\Phi=0 & (15-\mathrm{b})
\end{array}
$$

The vorticity is traditional defined as the curl of velocity; $\Omega=\nabla \times u$, and the dissipation function in the internal energy conservation equation, Eq. (15-b), is $\Phi=\lambda(\nabla \cdot u)^{2}$ $+\mu \operatorname{def}(u) \cdot \nabla u$.

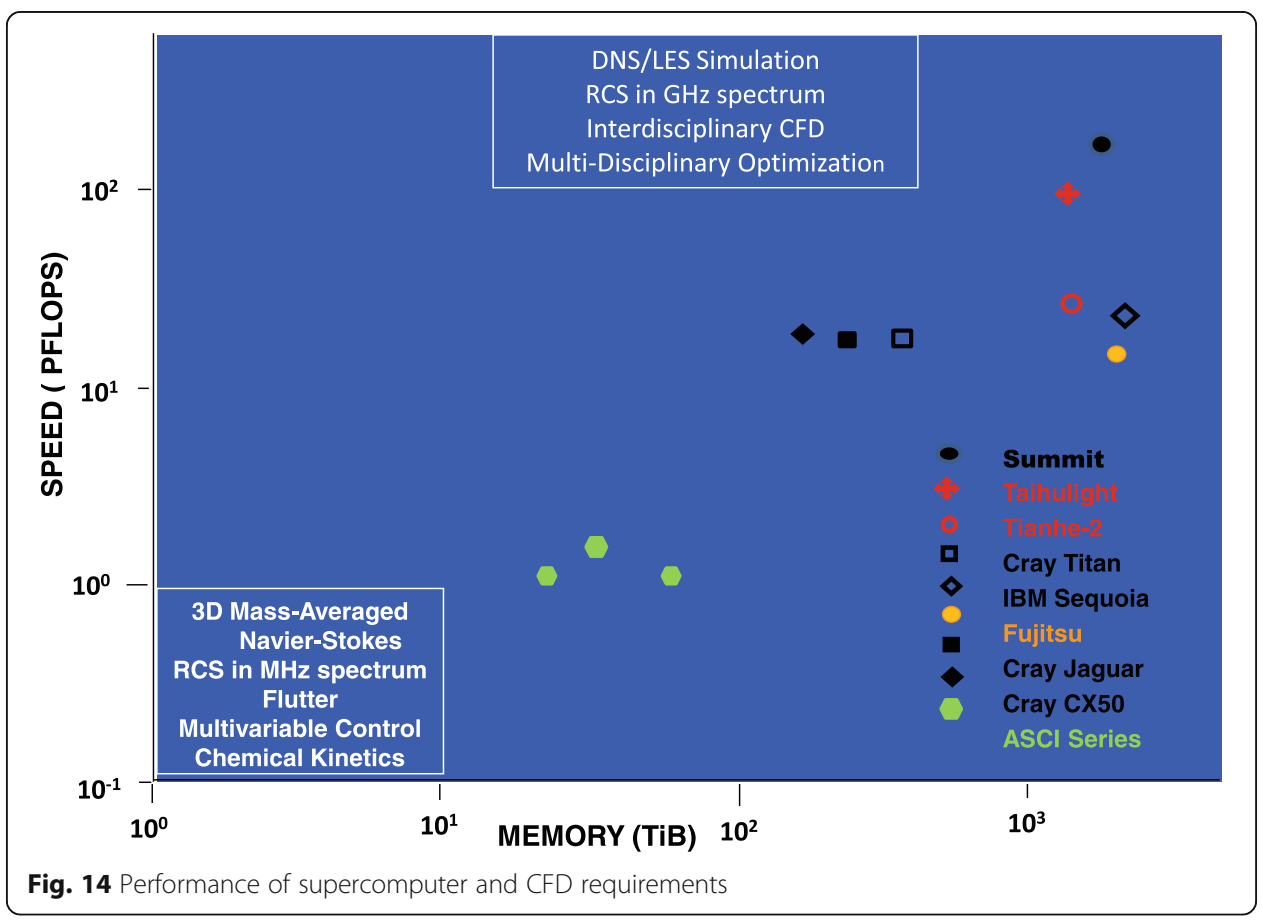


It is immediately recognized that the coupling between the vorticity dynamic equation and the energy conservation equation is only through the velocity component. In order to couple explicitly the two conservation laws with vorticity and velocity, the energy equation is split to get the kinetic energy transport equation as;

$$
\rho \partial(u \cdot u / 2) / \partial t+\rho u \cdot \nabla(u \cdot u / 2)+\nabla p \cdot u-(\nabla \cdot \tau) \cdot u=0
$$

Substitute Eq. (15-c) into Eq. (15-b), the conservation energy law in thermodynamic variables to get

$$
T \nabla S+u \times \Omega=\partial u / \partial t+\nabla h_{o}+\left[\nabla(\lambda \nabla \cdot u)+\nabla \mu\left(\nabla u+\nabla u^{T}\right)\right] / \rho+2 v \nabla(\nabla \cdot u)-v \nabla \times \Omega
$$

Equation (15-d) is the generalized Crocco's theorem for compressible flow. The Navier-Stokes equation in vorticity and velocity formulation, Eq. (15-a) and (15-c) is formerly closed by including the equation of state for the gas mixture and the constitute relationships of transport properties. The governing equations in vorticity-velocity formulation are completely consistent to the gas kinetic theory.

A few interesting physics of vortical dynamics can be distracted from Eq. (15-a) and (15-c): First, the forth term of Eq. (15-c), $(\Omega \cdot u) u$ has no counterpart in the equation of conservation of momentum, and it gives the vorticity dynamics a distinguish characteristic only existing in the three-dimensional flow field. In turbulent research its commonly referred as the vorticity stretching to become a critical property of turbulence, because the vorticity stretching is identically zero in two-dimensional field; $(\Omega \cdot u) u \equiv 0$. Second, in any vector field, there is a vector identity that is $\nabla \cdot(\nabla \times u)=\nabla \cdot \Omega \equiv 0$, which means the vorticity is a divergentless or divergent-free vector.

In an incompressible fluid medium, the governing equations, Eq. (15-a) and (15-c) degenerate into the classic results [85]

$$
\begin{array}{lr}
\partial \Omega / \partial t+(u \cdot \nabla) \Omega-(\Omega \cdot \nabla) u-v \nabla^{2} \Omega=0 & (15-\mathrm{e}) \\
T \nabla S+u \times \Omega+v \nabla \times \Omega=\partial u / \partial t+\nabla h_{o} & (15-\mathrm{f})
\end{array}
$$

For incompressible flow, the vorticity dynamic equation is linear and homogenous in $\Omega$, if the initial value of vorticity of flow field is null then the vorticity must be an invariant in time. It leads to the classic Helmholtz theorem that vorticity cannot be created nor destroyed in the interior of a homogeneous fluid and can be produced only on the interface boundaries. From Eq. (15-f), it is observed that only under the condition of constant enthalpy and a steady flow, the classic Crocco's theorem shows a unique relationship between entropy and vorticity [86].

From physical understanding, the Navier-Stoke equations in vorticity-velocity formulation shall be the most comprehensive approach for direct numerical simulation for turbulence. The physical fidelity is determined exclusively by the finest vorticity scales in time and space which are knew prior before the computational process. There are also known fact that the specification and implementation of boundary conditions for a wall-bound vorticity are difficult, but a physical compatible vorticity boundary condition can be derived from the non-slip velocity condition [87, 88]. In addition, the governing equation system is an incompletely parabolic system in the discrete space, and the well-established guidance for well-posed initial conditions and boundary values can be found in classic literatures [20]. 
The initial value and boundary conditions are paramount for computational simulation and are also problem dependent, thus must be remained as a research endeavor. The accomplishments by direct simulation for turbulence impact the basic understanding of fluid dynamics for future applications are invaluable.

On the challenge by expanding CFD beyond the realm of classic gas kinetic theory is based on the need to simulate the real gas effect in high-speed and high-enthalpy conditions. When the temperature is elevated above $1500 \mathrm{~K}$, the properties of air differ considerably from the perfect gas, because the excitations of internal structure of gas particle become significant. The internal degrees of freedom of molecule include the translational, rotational, vibrational, and electronic modes [4]. The electronic mode has also been referred to as nuclear excitation and can be further split into dissociation and ionization. All these internal excitations are quantized, and the bridge between the microstate of individual particle and macroscopic properties of gas mixture is linked by statistic mechanics through theories of probability and distribution functions. Only in equilibrium state, the internal energy distributions of a gas molecule or atom have been made easily understood by the quantum mechanics through the partition functions. Under the equilibrium states and from the statistic thermodynamics, the total energy of a molecule is additive and the partition functions on the other hand are factorized.

In other words, the energy of a molecule is measured above its zero-energy or ground state and is the sum of the energy of translational, rotational, vibrational, and electronic degrees of freedom. For atoms, the total energy includes only the translational and electronic modes

$$
e_{i}=e_{t}+e_{r}+e_{v}+e_{e} ; e_{i}=e_{t}+e_{e}
$$

The energy transfer by inelastic collisions is quantum restricted; the energy is transferred through discrete states and the transfer process is instantaneous because there is no continuity between quantum states. The sum of the internal energy by Eq. (16-a) is described individually by the partition function $Z$ from statistical thermodynamics which distributes the energy of particles among all the internal modes. Under the thermodynamic equilibrium condition, it is a function of specific volume $V$, and static temperature $T$;

$$
Z(V, T)=\sum_{i} g_{i} \exp \left(-e_{i} / \kappa T\right)
$$

The partition function describes a measure for a fraction of the total number of the $i^{\text {th }}$ molecules/atoms, of the system which possess an energy state of $e_{i}$. The symbol $g_{i}$ denotes the degeneracy which explicitly affirms how the molecule is formed. The partition function of a molecule is the product of all possible internal modes;

$$
Z(V, T)=Z_{t}(V, T) Z_{r}(V, T) Z_{v}(V, T) Z_{e}(V, T)
$$

From the partition functions, all thermodynamic properties of a system can be easily determined from statistics mechanics $[4,35]$. The factorization property of the partition functions is only valid so long as each energy mode can be assigned an energy level which is independent from the other energy modes. The schematic quantum spectrum of internal energy of a gas molecule is depicted in Fig. 15. The energy states of translation mode are so closely packed relative the datum; they are practically continuous, and contribute solely to the partial pressure of the gas mixture. All other internal degrees of freedom are clearly 


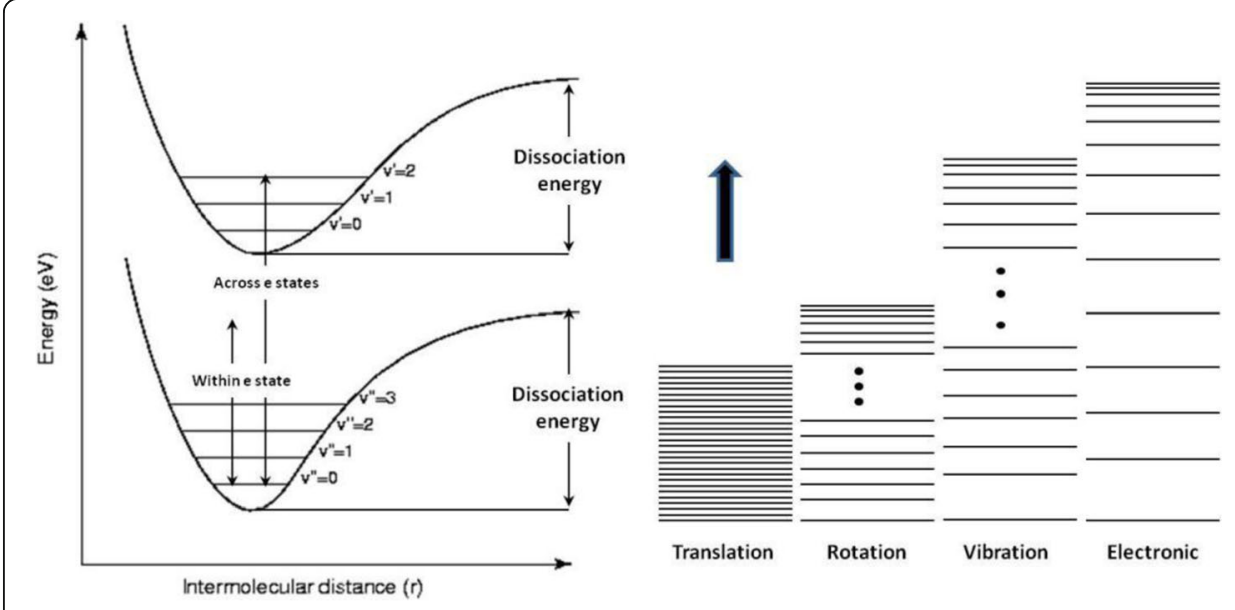

Fig. 15 Schematic quantum spectrum of internal energy of a gas molecule

distinctive quantum phenomena which are independent to the specific volume of the system thus do not contribute to the partial pressure of the gas mixture. In addition the rotational degree of freedom requires very few numbers of collisions to reach a fully excited state, for this reason, it is always considered to be equilibrated with the translation mode.

Although the actual transition process or the quantum jump is instantaneous, but to accumulate a sufficient energy to reach the next quantum state still requires a sufficient number of collisions to occur. The time lagging between jumps is often labelled as the relaxation phenomenon [89]. When the jump is limiting only to a next adjacent quantum state, the process is designated as the ladder-climbing process. Otherwise the transition over multiple quanta by a single collision and is known as the big-bang process.

In earth reentry simulations, the thermodynamic equilibrium conditions are not at all attainable; therefore the results by partition function are inapplicable. A wide range of approximations ether based on the principle of detail balance [90] or empirical data [73] are used to model the transitions of quantum mechanics. The energy exchange between translation and vibration, $Q_{t, v}$ in Eq. (14-c) is approximated by formulation of Landau and Teller;

$$
\begin{aligned}
& Q_{t, v}=\rho\left[e_{v}^{*}(t)-e_{v}\right] / \tau \\
& e_{v}^{*}(t)=R \Theta_{v} /\left(e^{\Theta_{v} / T}-1\right) ; \tau=k_{1} T^{5 / 6} e^{\left(k_{2} / T\right)^{1 / 3}} /\left[p\left(1-e^{-\Theta_{v} / T}\right)\right]
\end{aligned}
$$

where $\Theta_{v}$ and $\tau$ are the characteristic temperature and relaxation time scale of the vibration excitation transition. The energy transfer among vibration modes of different species is modelled by the empirical formula that derived from the experimental data collected and correlated by Millikan and White [89].

$$
\begin{aligned}
& Q_{t, v}=\rho\left[e_{v}^{*}(t)-e_{v}\right] / \tau \\
& e_{v}^{*}(t)=R \Theta_{v} /\left(e^{\Theta_{v} / T}-1\right) \\
& \tau=1.16 \times 10^{-3}\left[\left(m_{i}+m_{j}\right) / m_{i} m_{j}\right]^{1 / 2} \Theta_{v}^{4 / 3}\left[T^{-1 / 3}-0.15 m_{i} m_{j} /\left(m_{i}+m_{j}\right)-18.42\right]
\end{aligned}
$$

The energy transfer between vibration and electron excitation is often adopted the Treanor and Marlone model [91]. 


$$
Q_{e, v}=\sum_{i} \rho_{i}\left[\left(e_{t}-e_{v}\right) / \tau-\left(\bar{e}_{t, v}-e_{v}\right) / x_{i}\left(d n_{i} / d t\right)_{f}+\left(\bar{e}_{v, t}-e_{v}\right) / x_{i}\left(d n_{i} / d t\right)_{b}\right] \quad(17-\mathrm{c})
$$

Equation (17-c) is commonly referred to as the coupled vibration and dissociation (CVD) excitation model. In Eq. (17-c), the symbols $\bar{e}_{t, v}$ and $\bar{e}_{v, t}$ denote the average energy gained or lost during the quantum transition, and the notation $e_{v}$ is the vibration energy calculated from the Boltzmann distribution. There are a wide range of energy transition models between electron-ion collision by either the Coulomb logarithm formulation or other more recent estimates $[35,73,76,78]$. The total energy transfer between the quantum transitions that appeared in interdisciplinary governing equations; Eq. (14-c), (14-d), and (14-e) is actually the sum of each permissible mechanisms $Q_{v, \Sigma}$ $=Q_{t, v}+Q_{v, v}+Q_{e, v}$ and $Q_{e, \Sigma}=Q_{e, v}+Q_{e, i}$.

The approximate energy transfer by quantum transition among all internal degrees of freedom has often assumed that the quantum jumps follow the ladder-climbing process. The process can also be systematically determined from the population density distribution in a quantum state through the generation and the depletion process. The quantum jumps from translation-vibration, vibration-vibration, and vibration-dissociation transitions are describable by the changing vibrational quanta through inelastic collisions between diatomic molecules $M_{2}$ and the quantum states of $i, i, j$, and $j$;

$$
\begin{aligned}
& M_{2}(i)+M_{2} \rightleftarrows M_{2}\left(i^{\prime}\right)+M_{2} ; i^{\prime}=i \pm 1 \\
& M_{2}(i)+M_{2}(j) \rightleftarrows M_{2}\left(i^{\prime}\right)+M_{2}\left(j^{\prime}\right) ; i^{\prime}=i \pm 1, j^{\prime}=j \pm 1 \\
& M_{2}(i)+M_{2} \rightleftarrows 2 M+M_{2}
\end{aligned}
$$

The quantum jump is restricted to the immediate adjacent quantum level according to the Ladder-climbing process, and has been determined numerically by solving the master equation for population density distribution [92, 93];

$$
\begin{aligned}
d f / d t & =\sum_{i}\left[k_{v, t}\left(i \rightarrow i^{\prime}\right) f_{i} f-k_{v, t}\left(i^{\prime} \rightarrow i\right) f_{i} f\right] \\
& +\sum_{i, j}\left[k_{v, v}\left(i^{\prime}, j^{\prime} \rightarrow i, j\right) f_{i^{\prime}} f_{j^{\prime}}-k_{v, v}\left(i, j \rightarrow i^{\prime}, j^{\prime}\right) f_{i} f_{j}\right] \\
& +\sum_{i}\left[k_{v, d}(i \rightarrow \text { contiuum }) f_{M_{2}} f_{M^{-}}-k_{v, d}(\text { contimuum } \rightarrow i) f_{M} f_{M}\right]
\end{aligned}
$$

The modeling of quantum transition has served the engineering purposes for thermal protection for reentry space flights. All the models described by the forgoing discussions produced similar results from different numerical simulations [35, 76, 77], especially in the prediction of vibrational temperatures of oxygen and nitrogen molecules under the reentry conditions of the RAMC-II and Stardust probes.

A direct physical validation of the predicted nonequilibrium thermodynamic and chemical kinetics including quantum transitions with flight test data is presented by Fig. 16. The estimated error bar of the RAMC-II probe data covers a range of the peak-to-peak electron number density fluctuation from $3.0 \times 10^{11}$ to $1.2 \times 10^{12}$ due to the probe motion. The computational electron number densities under the chemically equilibrium and nonequilibrium models are depicted together with the validating flight test data at three altitudes along the reentry trajectory. The reentry speed decelerates from $7.66 \mathrm{~km} / \mathrm{s}$ to $7.65 \mathrm{~km} / \mathrm{s}$ from the altitudes from $81 \mathrm{~km}$ to $61 \mathrm{~km}$ and the ambient temperatures increased by 56.16 


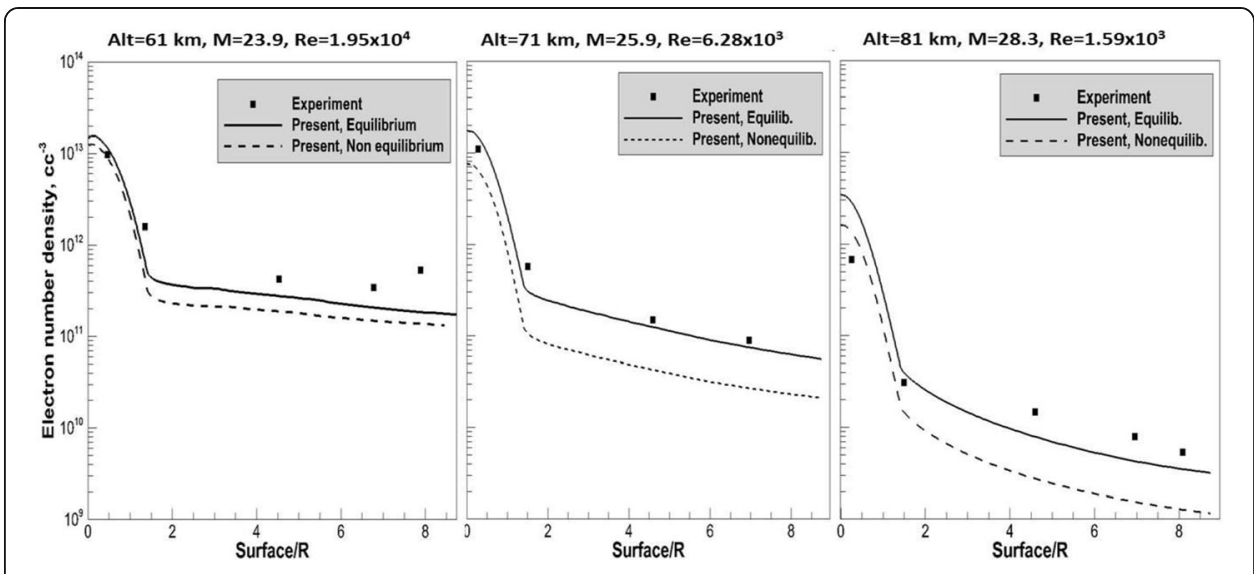

Fig. 16 verification of electron number density by flight data from RAMC-II probe

$\mathrm{K}$. It is anticipated that the nonequilibrium ionization occurs mostly in the stagnation region over the probe, and the equilibrium state shall be reached downstream and when the probe entered the denser atmosphere layer. However, there are large discrepancies among different chemical-physics models in predicted translational temperature within the shock layer. A detailed and incisive description of the quantum chemical-physics for basic understanding is still completely absented. The fundamental issue must be answered for basic understanding and to become another critical challenge to CFD.

A scientific breakthrough opportunity presents itself by employing the unprecedented high performance supercomputing capability to answer the formidable challenge through the ab initio (first principle) approach to quantum chemical-physics [94]. In other words, the nonequilibrium chemical reactions and energy transfer by the quantum transitions among internal excitations can be calculating directly from the chemical kinetics and quantum physics. The approach is based on the Born-Oppenheimer approximation by separating the wave function of the nuclei from the electron, in theory; the approximation is also applicable to molecule. The Schrodinger equation for a single electron is then solved for energy and wave function of an atom or a molecule, the required computational resource is truly prohibitive. From the most recent progress in molecular mechanics research, computations have been simplified by semi-empirical methods. In the ab initio computation process, the potential energy surface (PES) of atom/molecule structure becomes the center of computational quantum chemistry because the saddle point on the PES is closely related to the transition state [95]. The required topology knowledge has been developed by CFD for study surface shear pattern for three-dimensional separated flows. The complex topological formations of noddle, saddle, and focus singularities were established more than thirty years ago by the work of Tobak and Peaks [59].

The required research for interdisciplinary CFD involved quantum chemical-physics has clearly revealed that the pathfinding effort is unmistakably pointing to the need by returning to basic scientific research. These undertakings, no doubt are forbidden and arduous, but they also offer the undisputable scientific and technologic leadership worldwide.

\section{Concluding remarks}

From a glimpse of the remarkable CFD evolution over more than sixty years, one realizes that any scientific innovation for enriching human endeavor always reaches farther and 
thrives more vigorously by meeting the need of practical applications. The traditional CFD is established on the foundation of gas kinetic theory via a simplified elastic collision mechanism. And yet the critical aerodynamic bifurcations such as laminar-turbulent transition, turbulence, vortex breakdown, and dynamic stall are still beyond our reach. The barrier can be and shall be removed by embracing massive concurrent computational technique with the aid of high resolution algorithm development.

In order to expand and to increase our knowledge in basic science to meet future challenge for hypersonic flights and deep space explorations; research and developmental efforts must be focused on interdisciplinary simulation for selected critical technical areas. Only the knowledge that builds on the understanding of basic physical phenomena can further advance the modeling and simulation capability to attain the widest range of application.

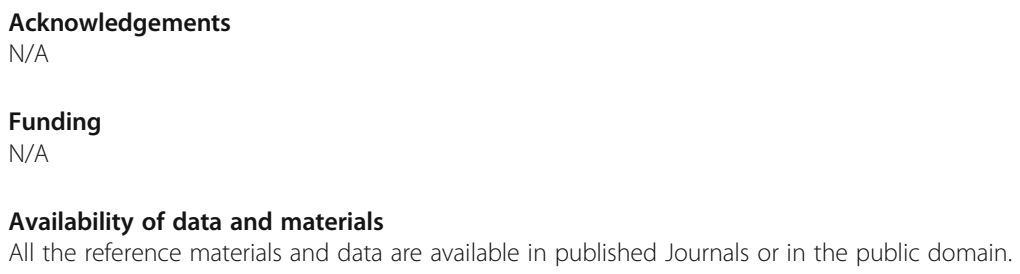

The article is the original work by the author exclusively for the Advance in Aerodynamics Journal as a scientific review for computational fluid dynamics. All authors read and approved the final manuscript.

\section{Competing interests}

There are no competition interests for the present endeavor.

\section{Publisher's Note}

Springer Nature remains neutral with regard to jurisdictional claims in published maps and institutional affiliations.

Received: 14 January 2019 Accepted: 14 January 2019

Published online: 31 January 2019

\section{References}

1. Navier M (1827) Memoire sur les Lois du Mouvement des Fluides. Mem De L'Acad D Sci 6:389

2. Stokes GG (1845) On the theories of the internal friction of fluid in motion. Trans Cambridge Phil Soc 8

3. Chapman S, Cowling TG (1964) The mathematical theory of non-uniform gases, 2nd edn. Cambridge Univ. Press

4. Clarke JF, McChesney M (1964) The dynamics of real gases. Butterworths, Washington

5. Hirschfelder JO, Curtiss CF, Bird BB (1954) Molecular theory of gases and liquids, 2nd edn. Wiley, New York

6. Capitelli M, Gorse C, Longo S, Giordano D (2000) Collision integrals of high-temperature air species. J Thermophysics Heat Transfer 14(2):259-268

7. Gustafasson B, Sandstrom A (1978) Incompletely parabolic problems in fluid dynamics. SIAM J Appl Math 35:343-335

8. Rizzi A, Inouye M (1973) Time-Split finite-volume method for three-dimensional blunt body flow. AlAA J 11:1478-1485

9. Thompson JF, Thames FC, Mastin CW (1974) Automatic numerical generation of body-fitted curvilinear coordinate system for field containing any number of arbitrary two-dimensional bodies. J Comput Phys 15:299-319

10. Harlow FH (1964) The particle-in-cell computing method for fluid dynamics, method in computational physics, vol 3, p 319

11. Shang JS, Surzhikov ST (2018) Plasma dynamics for aerospace engineering. Cambridge University Press, Cambridge, New York

12. Richardson LF (1910) The approximate arithmetical solution by definite differences of physical problems involving differential equations, with an application to the stresses in a masonry dam. Philos Trans Res Soc London, Series A 210: 307-357

13. Courant R, Friederichs KO, Lewy H (1928) Uber die partiellen differenzengleichungen der mathematischen physik. Mathenmatishe Annalen 100:32-74

14. Southwell RV (1940) Relaxation method in engineering science. Oxford University Press, London

15. Lax PD (1954) Weak solution of nonlinear hyperbolic equations and their numerical computation. Commun Pure Appl Math 7:159-163

16. Godunov SK (1959) Finite-difference method for numerical computational of discontinuous solution of the equations of fluid dynamics. Mat Sb 47:271-306

17. Anderson DA, Tannehill JC, Pletcher RH (1984) Computational fluid mechanics and heat transfer, 2nd edn. Taylor \&Francis, Bristol 
18. Peaceman DW, Rachford HH (1955) The numerical solution of parabolic and elliptic differential equations. J Soc Ind Appl Mat 3:28-41

19. Richtmyer RD, Morton KW (1967) Differential methods for initial-value problem, 2nd edn. Interscience Publishers, Wiley, New York

20. Shang JS (2009) Computational fluid dynamics application to aerospace science. Aeronaut J 113(1148)

21. Brandt A (1973) Multi-level adaptive technique (MALT) for fast numerical solution to boundary value problem, lecture notes in phys, vol 18. Springer-Verlag, Berlin, pp 82-89

22. Delaunay, B., Sur la Sphere Vide, Bull. Acad. Science, USSR, VII, Class. Sci. Mat. Nat. 793-800, 1934

23. Harten A (1983) High-resolution schemes for hyperbolic conservation Laws. J Comp Phys 49:375-385

24. Steger JL, Warming RF (1981) Flux vector splitting of the inviscid Gasdynamics equations with application to finite difference methods. J Comput Phys 40:263-293

25. Roe PL (1981) Approximate Riemann solvers, parameter vectors and difference schemes. J Comput Phys 43:357-372

26. Balsara D, Shu CW (2000) Monotonicity preserving weighted essentially non-oscillatory schemes with increasingly high order of accuracy. J Comp Physics 160:405-452

27. Shu CW, Osher S (1989) Implementation of essentially nonoscillatory shock capture scheme II. J Comp physics 83:32-78

28. Collatz $L$ (1966) The numerical treatment of differential equations. Springer-Verlag, New York, p 538

29. Lele SK (1992) Finite difference schemes with spectral-like resolution. J Comp Phys. 103:16-14

30. Gaitonde D, Shang JS (1997) Optimized compact-difference-based finite-volume schemes for linear wave phenomena. J Comp Physics 138:617-643

31. Gaitonde DV, Shang JS, Young JL (1999) Practical aspects of higher-order numerical schemes for wave propagation phenomena. Int J Numer Methods Eng 45:1849-1869

32. Korpriva D (1994) Multidomain spectral solution of compressible viscous flows. J Comp Physics 115:184-199

33. Korpriva D (1996) A conservative staggered-grid Chebyshev multi-domain method for compressible flows, II. A semistructure method. J Comp Physics 129:475-488

34. Carnahan B, Luther HA, Wilkes JO (1969) Applied numerical methods. Wiley, New York

35. Shang JS (2016) Computational electromagnetic-aerodynamics. Wiley, Hoboken

36. Katta VR, Roquemore WM (2008) Calculation of multidimensional flames using large chemical kinetics. AIAA J 46:16401650

37. Harten A, Enquist B, Osher S, Chakravarthy SR (1997) Uniformly high order accurate essentially non-oscillatory scheme III. J Comp Physics 131:3-47

38. Cockburn B, Shu CW (1989) TVD Runge-Kutta local projection discontinuous Galerkin finite element method for conservation laws II: general framework. Math Comp 52:411-435

39. Luo H, Baum JD, Lohner R (2008) Fast p-multigrid discontinuous Galerkin method for compressible flow at all speeds. J AIAA 46:635-652

40. Parsani M, Van den Abeele M, Lacor C, Turkel E (2010) Implicit LU-SGS algorithm for high-order methods on unstructured grid with p-multigrid strategy for solving the steady Navier-Stokes equations. J Comput Phys 229:828-850

41. Gao H, Wang ZJ, Huynh HT (2013) Differential formulation of discontinuous Galerkin and related methods for the Navier-Stokes equations. Commun Comp Phys 13:1013-1044

42. Leung TM, Zingg DW (2012) Aerodynamic shape optimization of wings using a parallel Newton-Krylov approach. AlAA J 50:540-550

43. Hirt CW (1969) Computer studied of time-dependent turbulent flows, high-speed computing in fluid dynamics, physics of fluid supplement II. American Inst. of phys, New York

44. Von Neumann J (1944) Proposal and analysis of a numerical method for the treatment of hydrodynamical shock problems. Nat Def Res Com Rept:AM-551

45. Taylor GI (1950) The formation of a blast wave by a very intense explosion. Proc Roy Soc London Series A 201:159-168

46. Chapman DR (1979) Computational aerodynamics development and outlook. AIAA J 17:1293-1313

47. MacCormack RW (1969) The effect of viscosity in hypervelocity impact cratering, AIAA preprint 1969-354, Cincinnati

48. Shang JS, Hankey WL (1977) Numerical solution of the compressible Navier-Stokes equations for a three-dimensional corner. AIAA J 15:1575-1582

49. II'yushin AA (1956) The law of plane section in the aerodynamics of high supersonic speeds. PMM 20:733-755

50. Dolling DS (2001) Fifty years of shock/boundary interaction: what next. AIAA J 39:1517-1531

51. Patankar SV, Pratap VS, Spalding DB (1974) Prediction of laminar flow and heat transfer in helically wield pipes. J Fluid Mech 62:539-551

52. Jameson A (1974) Iterative solution of transonic flows over airfoils and wings including flows at Mach 1. Commun Pure Appl Math 17:283-309

53. Murman EM, Cole JD (1971) Calculation of plane steady transonic flows. AIAA J 9:114-121

54. Levy JL (1978) Experimental and computational steady and unsteady transonic flow about a thick airfoil. AIAA J:564-572

55. Steger JL, Bailey HE, AIAA J (1980) Calculation of transonic aileron buzz. AIAA 18:249-255

56. Beam RM, Warming RF (1978) An implicit factored scheme for the compressible Navier-Stokes Equations. AIAA J 16:393401

57. Helliwell WS, Dickinson RP, Lubard SC (1981) Viscous flow over arbitrary geometries at high angle of attack. AIAA J 19(2): 191-197

58. Fasel $H(1976)$ Investigation of the instability of boundary layers by a finite-difference model of the Navier-Stokes equations. J Fluid Mech 72(2):355-383

59. Tobak M, Peaks DJ (1982) Topology of three-dimensional separated flows. Annu Rev Fluid Mech 14:61-85

60. Shang JS, Scherr SJ (1986) Navier-Stokes solution for a complete re-entry configuration. J Aircr 23:881-888

61. Barthelemy RR (1989) The National Aero-Space Plane program. AIAA:1989-5053

62. Curran ET (2001) Scramjet engines: the first forty years. J Propulsion Power 17:1138-1148

63. McClinton C, Bittner R, Kamath P (1990) CFD support to NASP design. AIAA:90-5249

64. Buning PG, Parks SJ, Chan WM, Renze KJ (1991) Application of the chimera overlapped grid scheme to simulation of space shuttle accent flows, proceedings of the 4th international symposium on CFD, Davis 
65. Sotnick JP, Kandula M (1994-1860) Buning P (1994) Navier-Stokes simulation of the space shuttle launch vehicle flight transonic flowfield using a large scale chimera grid system. AIAA

66. Gropp W, Lusk E, Skjellum A (1994) Using MPI: portable parallel programming with message passing interface. MIT Press, Cambridge

67. Shang JS, Wagner M, Pan Y, Blake DC (2000) Strategies for adopting FVTD on multicomputers. IEEE Comput Sci Eng 2(1): $10-21$

68. Bradshaw P (1994) Turbulence: the chief outstanding difficulty of our subject. Exp Fluids 16:203-216

69. Moin P, Mahesh K (1998) Direct numerical simulation; a tool in turbulent research. Annu Rev Fluid Mech 30:539-578

70. Piomelli U (1999) Large-eddy simulation: achievement and challenges. Prog Aerosp Sci 35:335-362

71. Smagorinsky JS (1963) General circulation experiments with the primitive equations. Mon Weather Rev 29:511-546

72. De Stafeno G, Goldstein DE, Vasilyev OV (2005) On the role of subgrid-scale coherent models of large-scale simulation. JFM 525:263-274

73. Park C (1989) Assessment of two-temperature kinetic model for ionizing air. J Thermophysics Heat Transfer 3:233-244

74. Candler GV, MacCormack RW (1991) Computation of weakly ionized hypersonic flows in thermochemical nonequilibrium. J Thermophysics 5:266-273

75. Josyula E, Shang JS (1991) Numerical study of hypersonic dissociated flow past blunt body. AIAA J 29(5):704-711

76. Olynick DR, Chen YK, Tauber ME (1999) Aerodynamics of the stardust sample return capsule. J Spacecraft and Rockets 36(3):442-462

77. Park C (2007) Calculation of stagnation-point heating rates associated with stardust vehicle. J Space and Rockets 44(1): 24-32

78. Surzhikov ST, Shang JS (2012) Coupled radiation-gasdynamic model for stardust earth entry simulation. J of Spacecraft and Rockets 49(5):875-888

79. Corke TC, Enloe CL, Wilkinson SP (2010) Plasma actuators for flow control. Annu Rev Fluid Mech 42:505-525

80. Starikovskaia SM (2006) Plasma assisted ignition and combustion, J. Phys. D. Appl Phys 39:R265-R299

81. Muller TJ, Kellogg JC, Ifja PG, Shkarayev SV (2006) Introduction to design of fixed-wing micro air vehicle. AIAA, New York

82. Jones KD, Bradshaw CJ, Papadopoulos J, Platz MF (2005) Bio-inspired design of flopping wing micro aerial vehicle. Aeronaut J 109(1098):385-393

83. Leibovich S (1978) The structure of vortex breakdown. Annu Rev Fluid Mech 10:221-246

84. Reshotko E (1976) Boundary-layer stability and transition. Annu Rev Fluid Mech 8:311-349

85. Tennekes H, Lumley JL (1972) A first course in turbulence. MIT Press, Cambridge

86. Liepmann HW, Roshotko A (1957) Elements of gasdynamics. Wiley, New York

87. Bhaunik S, Segapta TK (2015) A new velocity-vorticity formlation for direct numerical simulation of three-dimensional transitional and turbulent flows. J Comput Phys 284:230-260

88. Meitz RL, Fasel HF (2000) A compact-difference scheme for the Navier-Stokes equations in vorticity-velocity formulation. J Comput Phys 157:371-403

89. Millikan RC, White DR (1963) Systematics of vibrational relaxation. J Chem Phys 39(12):3209-3213

90. Ladau L, Teller E (1936) Zurtheorie der shallispersion. Physik Z Sowjetunion B 10:14

91. Treanor CE, Marrone PV (1962) Effect of dissociation on the rate of vibrational relaxation. Phys Fluids 5(9):1022-1026

92. Adamovich IV, Macheret SO, Rich JW, Treanor CE (1995) Vibrational relaxation and dissociation behind shock waves, part 2: master equation modeling. AIAA J 33:1070-1075

93. Josyula E, Bailey W, Suchyta C (2011) Dissociation modeling in hypersonic flows using state-to-state kinetics. J Thermophysics Heat Transfer 25(1):34-47

94. Lewars EG (2011) Computational Chemistry; Introduction to the theory and applications of molecule and quantum mechanics. Springer, New York

95. Eyring H, Walter J, Kimball GE (1944) Quantum chemistry. Wiley, New York

96. Gomez RJ (2011) 20+ years of CFD for space shuttle, NASA Johnson Space Center

\section{Submit your manuscript to a SpringerOpen ${ }^{\circ}$ journal and benefit from:}

- Convenient online submission

- Rigorous peer review

- Open access: articles freely available online

- High visibility within the field

- Retaining the copyright to your article

Submit your next manuscript at $\mathbf{s p r i n g e r o p e n . c o m ~}$ 